\title{
Local thermodynamical equilibrium and the $\beta$ frame for a quantum relativistic fluid
}

\author{
Francesco Becattini $^{1, \mathrm{a}}$, Leda Bucciantini ${ }^{2, \mathrm{~b}}$, Eduardo Grossi ${ }^{1, \mathrm{c}}$, Leonardo Tinti ${ }^{3, \mathrm{~d}}$ \\ ${ }^{1}$ Università di Firenze and INFN Sezione di Firenze, Florence, Italy \\ 2 Dipartimento di Fisica dell'Università di Pisa and INFN, 56127 Pisa, Italy \\ 3 Jan Kochanowski University, Kielce, Poland
}

Received: 27 October 2014 / Accepted: 31 March 2015 / Published online: 5 May 2015

(C) The Author(s) 2015. This article is published with open access at Springerlink.com

\begin{abstract}
We discuss the concept of local thermodynamical equilibrium in relativistic hydrodynamics in flat spacetime in a quantum statistical framework without an underlying kinetic description, suitable for strongly interacting fluids. We show that the appropriate definition of local equilibrium naturally leads to the introduction of a relativistic hydrodynamical frame in which the four-velocity vector is the one of a relativistic thermometer at equilibrium with the fluid, parallel to the inverse temperature four-vector $\beta$, which then becomes a primary quantity. We show that this frame is the most appropriate for the expansion of the stress-energy tensor from local thermodynamical equilibrium and that therein the local laws of thermodynamics take on their simplest form. We discuss the difference between the $\beta$ frame and Landau frame and present an instance where they differ.
\end{abstract}

\section{Introduction}

In recent years, relativistic hydrodynamics has drawn much attention. Part of the revived interest [1,2] is owing to the successful hydrodynamic description of the Quark-Gluon Plasma formed in collisions of nuclei at very high energy [3-10]. It is also known that hydrodynamics can be applied to a large portion of the phase diagram of condensed matter systems presenting quantum critical points [11-14]. Focusing on the quark-gluon plasma, close to the QCD critical temperature, the system is made of strongly interacting quantum fields and does not apparently allow for a description in terms of weakly interacting quasiparticles $[15,16]$. Thus, the use of kinetic theory to describe it can be questioned, and yet,

\footnotetext{
a e-mail: becattini@fi.infn.it

be-mail: leda.bucciantini@df.unipi.it

c e-mail: grossi@fi.infn.it

de-mail: dr.leonardo.tinti@gmail.com
}

because the microscopic interaction length is small compared to its overall size, the system is actually a fluid.

In principle, hydrodynamics does not need an underlying kinetic theory nor a discrete particle substratum, even if its use can be very effective to obtain useful relations [17]. Hydrodynamic is, in essence, the continuity equation of the mean value of the stress-energy tensor (and charge current) operator, which, being primarily expressed in terms of quantum fields, does not need a single-particle distribution function $f(x, p)$. In fact, its momentum-space integral expression in terms of $f(x, p)$ can be obtained under special conditions, those which make a kinetic approach suitable [18].

Consequently, all basic concepts in hydrodynamics should be defined independently of kinetic theory and of the singleparticle distribution function. Indeed, while flow velocity is usually defined as an eigenvector of some current, like in the Landau and Eckart frames, another very basic notion of hydrodynamics, that is, local thermodynamic equilibrium (LTE), is defined in most textbooks by means of kinetic theory; for instance, by making the collisional integral of the (relativistic) Boltzmann equation vanishing [18]. In fact, we will show in this work that this is not the most general definition; in quantum statistical mechanics LTE can be defined as a maximum of the entropy with specific constraints [19]. Furthermore it will be shown that, in the relativistic context, such a definition naturally leads to the introduction of a fourvector field-the inverse temperature four-vector $\beta$, which functions as a hydrodynamical velocity, giving rise to a new hydrodynamical frame other than the well-known Landau and Eckart frame.

Thus far, this four-vector field has been mostly considered as a secondary quantity, formed by multiplying the invariant temperature $1 / T$ by an otherwise defined velocity fourvector $u$. Recently, Van and Biro [20] have argued that $\beta$ in fact defines a new independent frame, a conclusion that we fully support. Indeed, in this paper, we will reinforce it 
and demonstrate it in the most general quantum relativistic framework without resorting to kinetic arguments. We will show that it is much more natural and convenient to take $\beta$ as a primordial field related to the concept of LTE, so that the four-velocity of a relativistic fluid can be defined starting from the $\beta$ field and not vice versa:

$u(x) \equiv \frac{\beta}{\sqrt{\beta^{2}}}$.

The paper is organized as follows. In Sects. 2,3 we review the concept of local thermodynamical equilibrium in relativistic quantum statistical mechanics and introduce the $\beta$ frame. In Sect. 4 we will show how to operationally define the $\beta$ vector through an ideal relativistic thermometer, providing better insight into its physical meaning. In Sect. 5 we will discuss the form of the stress-energy tensor in the $\beta$ frame, in Sect. 6 we will point out the difference between the $\beta$ and Landau frames; finally in Sect. 7 we will discuss the separation between the ideal and dissipative part of the stressenergy tensor.

\section{Notation}

In this paper we use the natural units, with $\hbar=c=K=1$.

The Minkowskian metric tensor is $\operatorname{diag}(1,-1,-1,-1)$; for the Levi-Civita symbol we use the convention $\epsilon^{0123}=1$.

We will use the relativistic notation with repeated indices assumed to be saturated, however, contractions of indices will be sometimes denoted with dots, e.g. $u \cdot T \cdot u \equiv$ $u_{\mu} T^{\mu v} u_{\nu}$. Operators in Hilbert space will be denoted by a large upper hat, e.g. $\widehat{R}$ while unit vectors will be denoted with a small upper hat, e.g. $\hat{v}$. We will work with a symmetric stress-energy tensor with an associated vanishing spin tensor.

\section{Local thermodynamical equilibrium in relativistic quantum statistical mechanics}

In the most general framework of quantum statistical mechanics, LTE is defined by the maximization of the Von Neumann entropy $S=-\operatorname{tr}(\widehat{\rho} \log \widehat{\rho}), \widehat{\rho}$ being the density operator, with the constraints of fixed densities of energy, momentum, and charge [19]. As has been mentioned in the Introduction, such a definition does not require any underlying kinetic theory; the only requirement is that densities significantly vary over distances much larger than the typical microscopic scale.

In non-relativistic thermodynamics, the LTE definition is an unambiguous one and leads to a unique density operator obtained by maximizing the function of $\widehat{\rho}$, with $\operatorname{tr} \widehat{\rho}=1$, for any time $t$,

$$
\begin{aligned}
& -\operatorname{tr}(\widehat{\rho} \log \widehat{\rho})+\int \mathrm{d}^{3} \mathbf{x} b(\mathbf{x}, t)[\langle\widehat{h}(\mathbf{x}, t)\rangle-h(\mathbf{x}, t)] \\
& -b(\mathbf{x}, t) \mathbf{v}(\mathbf{x}, t) \cdot[\langle\widehat{\boldsymbol{\pi}}(\mathbf{x}, t)\rangle-\boldsymbol{\pi}(\mathbf{x}, t)] \\
& -\xi(\mathbf{x}, t)[\langle\widehat{q}(\mathbf{x}, t)\rangle-q(\mathbf{x}, t)],
\end{aligned}
$$

where $h, \pi$, and $q$ are the actual values of the energy, momentum and particle (or charge) density, respectively; $b=1 / T$ and $\xi=\mu / T$ are point-dependent Lagrange multipliers, as well as $\mathbf{v}$ whose meaning is the mean velocity of the particles. The symbol \langle\rangle stands for the renormalized mean value of the operators:

$$
\langle\widehat{A}\rangle=\operatorname{tr}(\widehat{\rho} \widehat{A})_{\text {ren }} .
$$

For the simple case of a free quantum field theory this corresponds to the normal ordering of creation and destruction operator and, if $\widehat{A}$ is quadratic in the fields, to the subtraction of its vacuum expectation value:

$\operatorname{tr}(\widehat{\rho} \widehat{A})_{\text {ren }}=\operatorname{tr}(\widehat{\rho}: \widehat{A}:)=\operatorname{tr}(\widehat{\rho} \widehat{A})-\langle 0|\widehat{A}| 0\rangle$.

The density operator $\widehat{\rho}_{\mathrm{LE}}$ resulting from the maximization of (1) is called the LTE density operator:

$$
\begin{aligned}
\widehat{\rho}_{\mathrm{LE}}= & \frac{1}{Z_{\mathrm{LE}}} \exp \left[-\int \mathrm{d}^{3} \mathrm{x} b(\mathbf{x}, t) \widehat{h}(\mathbf{x}, t)\right. \\
& +b(\mathbf{x}, t) \mathbf{v}(\mathbf{x}, t) \cdot \widehat{\boldsymbol{\pi}}(\mathbf{x}, t)-\xi(\mathbf{x}, t) \widehat{q}(\mathbf{x}, t)],
\end{aligned}
$$

where $Z_{\mathrm{LE}}$ is the normalizing factor making $\operatorname{tr} \widehat{\rho}_{\mathrm{LE}}=1$. The values of the Lagrange multipliers $b, \mathbf{v}$, and $\xi$ are obtained enforcing $\langle\hat{A}\rangle=A$, where $A$ is respectively the actual value of the energy, momentum and charge of the system. A Galilean transformation does not change the resulting density operator except for a shift of the parameter $\mathbf{v}$, but the entropy $S=-\operatorname{tr}\left(\widehat{\rho}_{\mathrm{LE}} \log \widehat{\rho}_{\mathrm{LE}}\right)$ is invariant. It should be emphasized that $\widehat{\rho}_{\mathrm{LE}}$ is not the true density operator. Indeed, in the Heisenberg picture the LTE density operator $\widehat{\rho}_{\mathrm{LE}}$ in Eq. (2) is explicitly dependent on time through the time dependence of the operators, while the true density operator must be time-independent in the Heisenberg picture. The relation between the true density operator $\widehat{\rho}$ and $\widehat{\rho}_{\text {LE }}$ will be discussed in Sect. 5.

Extending the definition of LTE to quantum relativistic statistical mechanics in flat spacetime is not straightforward because energy density and momentum density are framedependent quantities in a much stronger fashion than in nonrelativistic mechanics. To make it fully covariant, it is necessary to fix a $\tau$-parametric family of spacelike hypersurfaces $\Sigma(\tau)$. The timelike unit vector field $n(x)$ normal to the surfaces defines the world lines of observers (see Fig. 1), yet the parameter $\tau$, in general, does not coincide with the proper time of comoving clocks. As is well known, for orthogonal surfaces to exist, the field $n(x)$ must be vorticity free, i.e. it ought to fulfill the equation: 


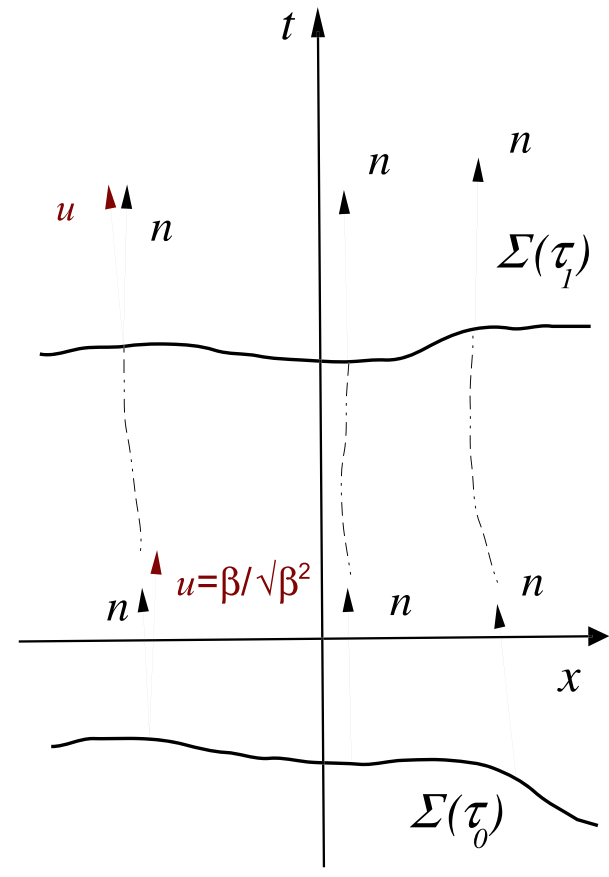

Fig. 1 Spacelike hypersurfaces $\Sigma(\tau)$ and their normal versor $n$ defining local thermodynamical equilibrium for a relativistic fluid in Minkwoski spacetime. If the $\beta$ field has vanishing vorticity, $\beta$ can be chosen parallel to $n$ at each point; conversely, the normal versor and the $\beta$ direction do not coincide (see Sect. 3)

$\epsilon_{\mu \nu \rho \sigma} n^{\nu}\left(\partial^{\rho} n^{\sigma}-\partial^{\sigma} n^{\rho}\right)=0$.

For the comoving frame having $n$ as time direction, we enforce the mean energy-momentum and charge density to be the actual ones everywhere:

$n_{\mu} \operatorname{tr}\left(\widehat{\rho}_{\mathrm{LE}} \widehat{T}^{\mu v}(x)\right)_{\mathrm{ren}}=n_{\mu}\left\langle\widehat{T}^{\mu v}(x)\right\rangle_{\mathrm{LE}} \equiv n_{\mu} T_{\mathrm{LE}}^{\mu \nu}(x)=n_{\mu} T^{\mu \nu}(x)$

$n_{\mu} \operatorname{tr}\left(\widehat{\rho}_{\mathrm{LE}} \widehat{j}^{\mu}(x)\right)_{\mathrm{ren}}=n_{\mu}\left\langle\widehat{j}^{\mu}(x)\right\rangle_{\mathrm{LE}} \equiv n_{\mu} j_{\mathrm{LE}}^{\mu}(x)=n_{\mu} j^{\mu}(x)$

where $\widehat{T}$ is the stress-energy tensor operator and $\widehat{j}$ the conserved current (if any). The function to be maximized as a function of $\widehat{\rho}$, with $\operatorname{tr} \widehat{\rho}=1$, at any $\tau$, reads

$$
\begin{aligned}
& -\operatorname{tr}(\widehat{\rho} \log \widehat{\rho})+\int_{\Sigma(\tau)} \mathrm{d} \Sigma n_{\mu}\left[\left(\left\langle\widehat{T}^{\mu \nu}(x)\right\rangle-T^{\mu \nu}(x)\right) \beta_{\nu}(x)\right. \\
& \left.-\left(\left\langle\widehat{j}^{\mu}(x)\right\rangle-j^{\mu}(x)\right) \xi(x)\right]
\end{aligned}
$$

where $d \Sigma$ is the measure (in the Minkowski spacetime) of the hypersurface, $\beta$ is, by definition, the inverse temperature four-vector, and $\xi$ a scalar field of Lagrange multipliers whose meaning will be made clear shortly. The solution is

$\widehat{\rho}_{\mathrm{LE}}=\frac{1}{Z_{\mathrm{LE}}} \exp \left[-\int_{\Sigma(\tau)} \mathrm{d} \Sigma n_{\mu}\left(\widehat{T}^{\mu v}(x) \beta_{\nu}(x)-\xi(x) \widehat{j}^{\mu}(x)\right)\right]$.

This covariant form of a local equilibrium operator was, to our knowledge, first obtained with this variational method by
Zubarev [21]. It is clear that the operator in (5) does depend on the particular hypersurface $\Sigma(\tau)$ (whence on the field $n$ ). Accordingly, the mean values $T_{\mathrm{LE}}$ and $j_{\mathrm{LE}}$ depend on the hypersurface $\Sigma$, hence, in general one can write

$T_{\mathrm{LE}}^{\mu v}=T_{\mathrm{LE}}^{\mu v}[\beta, \xi, n] \quad j_{\mathrm{LE}}^{\mu}=j_{\mathrm{LE}}^{\mu}[\beta, \xi, n]$,

so that even the $\beta$ field, obtained as a solution of the Eq. (4), will depend on $n$ :

$n_{\mu} T_{\mathrm{LE}}^{\mu v}[\beta, \xi, n]=n_{\mu} T^{\mu v} \quad n_{\mu} j_{\mathrm{LE}}^{\mu}[\beta, \xi, n]=n_{\mu} j^{\mu}$,

where the square brackets mean that the dependence of the currents on the fields $\beta, \xi$ and $n$ is in general functional (e.g. there could be a dependence on the derivatives). For all means to be independent of it, the divergence of the integrand should vanish - provided that some boundary conditions are enforced [22] — a condition which is met if

$\partial_{\mu} \beta_{\nu}+\partial_{\nu} \beta_{\mu}=0 \quad \partial_{\mu} \xi=0$,

whose solution is (see also Ref. [22]):

$\beta_{v}=b_{v}+\varpi_{\nu \lambda} x^{\lambda} \quad \xi=\mathrm{const}$,

whence

$\varpi_{\nu \lambda}=-\frac{1}{2}\left(\partial_{\nu} \beta_{\lambda}-\partial_{\lambda} \beta_{\nu}\right)$.

The above equations just define the well-known condition of global thermodynamical equilibrium (GTE) for a relativistic fluid: $\beta$ must be a Killing vector [23]. They ensure the stationarity of the density operator, which now reads [from (5)]

$$
\begin{aligned}
\widehat{\rho} & =\frac{1}{Z} \exp \left[-b_{\nu} \widehat{P}^{v}+\frac{1}{2} \varpi_{\lambda v} \widehat{J}^{\lambda v}+\xi \widehat{Q}\right] \\
& =\frac{1}{Z} \exp \left[-\beta_{v}(x) \widehat{P}^{v}+\frac{1}{2} \varpi_{\lambda \nu} \widehat{J}_{x}^{\lambda v}+\xi \widehat{Q}\right]
\end{aligned}
$$

where, in the rightmost expression, we have taken advantage of the translated angular momentum operator:

$\widehat{J}^{\lambda v}=\widehat{J}_{x}^{\lambda v}+x^{\lambda} \widehat{P}^{v}-x^{\nu} \widehat{P}^{\lambda}$.

The general GTE form (10) depends-besides the chemical potential - on 10 constant parameters, as many as the generators of the Poincaré group. The density operator (10) comprises all known instances of GTE in Minkowski spacetime including the rotating equilibrium, which will be further discussed in Sect. (6).

Going back to local equilibrium, as long as the field $n(x)$ is not specified, the definition of LTE is ambiguous. ${ }^{1}$ To show that there is a preferential choice thereof, one can calculate the total entropy by using (2)

\footnotetext{
${ }^{1}$ Note that the field $n$ does not necessarily coincide with the hydrodynamic velocity field $u$ albeit, as we will see, it is related to it.
} 


$$
\begin{aligned}
S= & -\operatorname{tr}\left(\widehat{\rho}_{\mathrm{LE}} \log \widehat{\rho}_{\mathrm{LE}}\right)_{\mathrm{ren}}=\log Z_{\mathrm{LE}} \\
& +\int_{\Sigma(\tau)} \mathrm{d} \Sigma n_{\mu}\left(T_{\mathrm{LE}}^{\mu \nu} \beta_{v}-\xi j_{\mathrm{LE}}^{\mu}\right) .
\end{aligned}
$$

A crucial and mostly unspoken assumption in relativistic extension of thermodynamics is that $\log Z_{\mathrm{LE}}$ can be written as an integral over the hypersurface $\Sigma$ of a four-vector field, defined as thermodynamical potential current $\phi^{\mu}$, depending on the functions $\beta$ and $\xi$,

$$
\begin{aligned}
\log Z_{\mathrm{LE}}(\tau) & =\log \operatorname{tr}\left(\exp \left[-\int_{\Sigma(\tau)} \mathrm{d} \Sigma n_{\mu}\left(\widehat{T}^{\mu v} \beta_{\nu}-\xi \widehat{j}^{\mu}\right)\right]\right) \\
& =\int_{\Sigma(\tau)} \mathrm{d} \Sigma n_{\mu} \phi^{\mu}[\beta, \xi, n] .
\end{aligned}
$$

This assumption is necessary for the existence of an entropy current $s^{\mu}$ which is one of the starting points of Israel's formulation of relativistic hydrodynamics $[24,25]$. Although (13) should be proved, we account it in this work as an ansatz. Hence, in view of (12) and (13), the entropy current reads

$s^{\mu}=\phi^{\mu}+T_{\mathrm{LE}}^{\mu v} \beta_{v}-\xi j_{\mathrm{LE}}^{\mu}+s_{T}^{\mu}(n)$

where $s_{T}(n)$ is an arbitrary four-vector field orthogonal to $n$. Note from (13) that also $\phi$ is defined up to an arbitrary four-vector field orthogonal to $n$. It should be emphasized that in non-equilibrium situations, since $\partial_{\mu} s^{\mu} \neq 0$, the total entropy $S$ in (12) is a frame-dependent quantity, as it varies with the integration hypersurface $\Sigma$. Indeed, like $T_{\mathrm{LE}}$ and $j_{\mathrm{LE}}$, the local current $\phi$ will also depend on the hypersurface $\Sigma$ (see its dependence on $n$ in Eq. 13).

A great simplification would be achieved if $n=\hat{\beta}=$ $\beta_{\mu} / \sqrt{\beta^{2}}$, as the number of independent variables on which mean values depend would be reduced. With this choice Eq. (4) would become

$\beta_{\mu} T_{\mathrm{LE}}^{\mu v}[\beta, \xi]=\beta_{\mu} T^{\mu v} \quad \beta_{\mu} j_{\mathrm{LE}}^{\mu}[\beta, \xi]=\beta_{\mu} j^{\mu}$,

where the right hand sides contain the true values at each point. This choice is what we define as a $\beta$ frame, with a fluid velocity defined as

$u(x) \equiv \frac{\beta}{\sqrt{\beta^{2}}}$.

Indeed, setting $n=\hat{\beta}$ is possible only if the $\beta$ field solution of Eq. (6), also fulfills Eq. (3). In fact, this equation does not apply even for the simple case of a rigid velocity field, which is actually a global thermodynamical equilibrium one (see Appendix A). Notwithstanding, also in the vortex case, it is possible to find a proper definition of the $n$ field based on $\beta$, as will be shown in the next section.

From a physical viewpoint, the $\beta$ frame is identified by the four-velocity of a relativistic thermometer at local equilibrium with the system, what will be discussed in detail in Sect. 4. This frame has more peculiar features. As an example, let us contract Eq. (14) with $n_{\mu}$, which enables us to use
Eq. (4) to replace the local equilibrium averages $T$ and $j$ with their actual values

$s^{\mu} n_{\mu}=n_{\mu} \phi^{\mu}+n_{\mu} T^{\mu v} \beta_{\nu}-\xi n_{\mu} j^{\mu}$.

The left hand side is the entropy density seen by the observer moving with four-velocity $n$. If $n_{\mu}=\hat{\beta}_{\mu}$, the Eq. (16) is manifestly the basic relation of thermodynamics expressing the proper entropy density $s$ as a function of proper energy and charge density

$\sqrt{\beta^{2}} s=\beta \cdot \phi+\beta_{\mu} \beta_{v} T^{\mu v}-\xi \beta_{\mu} j^{\mu}$,

provided that $\beta^{2}=1 / T^{2}$ and $\xi=\mu / T$, what makes the physical meaning of $\beta$ and $\xi$ apparent. Indeed, introducing the symbols $\rho$ for the proper energy density and $q$ for the proper charge density,

$T s=T^{2} \beta \cdot \phi+\rho-\mu q$,

where

$\rho=\frac{\beta_{\mu} \beta_{\nu} T^{\mu \nu}}{\beta^{2}} q=\frac{\beta_{\mu} j^{\mu}}{\sqrt{\beta^{2}}}$.

Equation (18) tells us that the $\beta$ frame is the one where the basic thermodynamic relation between proper entropy density and proper (true) energy and charge densities takes on its simplest form. In different frames, this relation is to be obtained contracting with a vector different from $n$ and it may thus contain additional terms, most likely of the second order in the derivatives (see discussion in Appendix B). We conclude this section by noting that in the familiar global thermodynamical equilibrium, it is well known [22,24,25] that the four-vector field $\phi^{\mu}=p \beta^{\mu}$ where $p$ is the pressure, hence the (18) can be written in the more familiar form

$T s=p+\rho-\mu n$.

In fact, at local thermodynamical equilibrium, the thermodynamic potential current $\phi$ may have additional terms depending, e.g., on derivatives of the $\beta$ and $\xi$ fields. If these additional terms do have a longitudinal (along $\beta$ ) component, then the above equation is to be replaced by the most general (18).

\section{Local thermodynamical equilibrium for a general $\beta$ field}

For a general, non-vorticity-free field $\beta$, the identification $n=\hat{\beta}$ is not possible and must be modified. One can iteratively construct a field $n(x)$ which fulfills Eq. (3) and, at the same time, reproduce the well-known features of global thermodynamical equilibrium with rotation (see the discussion in $[22,26])$. Take 
$b_{\mu}^{(1)}=\beta_{\mu}+\frac{1}{2} x^{\nu}\left(\partial_{\mu} \beta_{\nu}-\partial_{\nu} \beta_{\mu}\right)$.

Clearly, $\partial_{\mu} b_{\nu}^{(1)}-\partial_{\nu} b_{\mu}^{(1)} \approx O\left(\partial^{2}\right)$. Iteratively, one can add to $b_{\mu}^{(1)}$ higher-order derivative terms which are antisymmetric in $\mu \nu$ to eliminate gradients at some order. For instance,

$b_{\mu}^{(2)}=\beta_{\mu}+x^{\nu}\left(1+\frac{x \cdot \partial}{3}\right)\left[\frac{1}{2}\left(\partial_{\mu} \beta_{\nu}-\partial_{\nu} \beta_{\mu}\right)\right]$

implies $\partial_{\mu} b_{\nu}^{(2)}-\partial_{\nu} b_{\mu}^{(2)} \approx \mathcal{O}\left(\partial^{3}\right)$. Thereby, we can construct a field $b(x)$ with vanishing external derivative,

$\partial_{\mu} b_{\nu}-\partial_{\nu} b_{\mu}=0$,

which can be used to define the LTE hypersurfaces $\Sigma(\tau)$, i.e.,

$n(x) \equiv \hat{b}(x)$,

because, as is apparent, if $b$ fulfills Eq. (3), any field collinear to it will. Hence, from Eqs. (21)-(22) we generalize to all orders, defining $\varpi$ :

$\beta_{\nu}(x) \equiv b_{v}(x)+\varpi_{\nu \lambda}(x) x^{\lambda}$,

with

$$
\begin{aligned}
\varpi_{\nu \lambda}(x)= & -\frac{1}{2}\left(\partial_{\nu} \beta_{\lambda}-\partial_{\lambda} \beta_{\nu}\right) \\
& -\frac{1}{6}\left(x^{\rho} \partial_{\rho} \partial_{\nu} \beta_{\lambda}-x^{\rho} \partial_{\rho} \partial_{\lambda} \beta_{\nu}\right)+\cdots
\end{aligned}
$$

It is apparent that the obtained expressions (24) and (25) are in full agreement with the global equilibrium case (8) and (9), respectively.

With the same LTE density operator as in Eq. (5), the field $\beta$ is now the solution of a modified version of the Eq. (15) enforcing the equality of the mean-energy and momentum density,

$$
\begin{aligned}
b_{\mu} T_{\mathrm{LE}}^{\mu \nu}[\beta, \xi] & =b_{\mu} T^{\mu \nu} \Longrightarrow\left(\beta_{\mu}-\varpi_{\mu \lambda}[\beta] x^{\lambda}\right) T_{\mathrm{LE}}^{\mu v}[\beta, \xi] \\
& =\left(\beta_{\mu}-\varpi_{\mu \lambda}[\beta] x^{\lambda}\right) T^{\mu \nu} \\
b_{\mu} j_{\mathrm{LE}}^{\mu}[\beta, \xi] & =b_{\mu} j^{\mu} \Longrightarrow\left(\beta_{\mu}-\varpi_{\mu \lambda}[\beta] x^{\lambda}\right) j_{\mathrm{LE}}^{\mu}[\beta, \xi] \\
& =\left(\beta_{\mu}-\varpi_{\mu \lambda}[\beta] x^{\lambda}\right) j^{\mu}
\end{aligned}
$$

with $\varpi$ given by (25). We stress that, for a vortex $\beta$ field, it is not possible to restore Eq. (15) instead of (26) to determine $\beta$, for $T_{\mathrm{LE}}$ requires a LTE density operator (5) to be defined and this in turn demands the constraints in the specific form (4) with $n$ being vorticity free.

By using (5) and (13), one can find an expression of the entropy current,

$s^{\mu}=\phi^{\mu}+T_{\mathrm{LE}}^{\mu v} \beta_{v}-\xi j_{\mathrm{LE}}^{\mu}+s_{T}^{\mu}(n)$.

The entropy density in the local rest frame of the fluid is obtained by contracting (27) with $\beta$ :

$$
\sqrt{\beta^{2}} s=\phi^{\mu} \beta_{\mu}+\beta_{\mu} T_{\mathrm{LE}}^{\mu v} \beta_{\nu}-\xi \beta_{\mu} j_{\mathrm{LE}}^{\mu}+\beta \cdot s_{T}(n) .
$$

However, unlike in the non-vortex case, replacing the local equilibrium mean values with the true ones is not straightforward.

\section{Temperature and thermometers in relativity}

So far, we have defined the temperature (and four-velocity) in a local equilibrium state as a Lagrange multiplier in the constrained (with fixed energy and momentum densities) maximization of the entropy. This mathematical definition corresponds to a more physical one which can be obtained by introducing the notion of an ideal relativistic thermometer. Just as in classical thermodynamics, this is, by definition, a "small" object able to instantaneously achieve thermodynamical equilibrium with the system in contact with it. Besides, it should have some macroscopic internal property (such as size, resistivity etc.) which varies as a function of temperature, so that it can be used to define a scale thereof.

In the relativistic context, an ideal thermometer can exchange both energy and momentum with the system, and therefore its response is not limited to a change of its internal property gauging the temperature, but it also includes a change of its four-velocity. In other words, once in contact with the system, the idealized relativistic thermometer will move at some finite speed which is determined by the local equilibrium conditions. Now, the discussion gets easier considering both the system and the thermometer small yet finite. If the thermometer attains full thermodynamical equilibrium with the system, the entropy will be maximal with respect to energy and momentum exchange; thus we can write (the subscript $T$ refers to the thermometer quantities)

$\frac{\partial S}{\partial P^{\mu}}=\frac{\partial S_{T}}{\partial P_{T}^{\mu}}$,

keeping the proper volumes and the conserved charges fixed. Now, let us suppose that the system is so small that $\beta$ and $\xi$ are essentially constant over the system and thermometer volumes so as to take them out of the integral sign in Eq. (12), ${ }^{2}$

$$
\begin{aligned}
S= & \log Z_{\mathrm{LE}}+\int \mathrm{d} \Sigma_{\mu}\left(T^{\mu \nu} \beta_{\nu}-\xi j^{\mu}\right) \simeq \log Z_{\mathrm{LE}} \\
& +\beta_{\nu} \int \mathrm{d} \Sigma_{\mu} T^{\mu \nu}-\xi \int \mathrm{d} \Sigma_{\mu} j^{\mu}=\log Z_{\mathrm{LE}}+\beta_{\nu} P^{\nu}-\xi Q
\end{aligned}
$$

where we have used the (4). Note that $P$ and $Q$ do not depend on the frame because the divergences of $T$ and $j$ are assumed to vanish (the interaction energy between system and thermometer is negligible by assumption). Hence, according to Eq. (28) and keeping in mind the basic relations of the equilibrium relativistic thermodynamics which express the mean

\footnotetext{
${ }^{2}$ Henceforth, we will use the shorthand $\mathrm{d} \Sigma_{\mu}$ for $\mathrm{d} \Sigma n_{\mu}$.
} 
values of energy-momentum as derivatives of $\log Z_{\mathrm{LE}}$, we obtain

$\beta_{v}=\beta_{\nu \mathrm{T}}$

The above equation implies that a relativistic thermometer in thermodynamical equilibrium with the system will mark the temperature $T_{0}=/ \sqrt{\beta^{2}}$ and move with a speed $\beta / \sqrt{\beta^{2}}$. In this case, the thermometer is defined as comoving and the marked temperature is generally referred to as the local temperature.

Alternatively, one can retain a more traditional definition of an ideal thermometer as a "small" object endowed with a temperature gauge and able to instantaneously achieve thermodynamical equilibrium with the system in contact with it with respect to energy exchange; its velocity $v$ can be externally imposed. According to the generally accepted extension of thermodynamics to relativity $[27,28]$, one has to choose the frame where the thermometer is at rest and therein enforce the condition of maximal entropy with respect to only energy exchange,

$\frac{\partial S}{\partial E}=\frac{\partial S_{T}}{\partial E_{T}}$

which results in the equality of the time components of the $\beta$ vectors in that frame:

$\beta^{0}=\beta_{T}^{0}$

or

$\beta \cdot v=\frac{1}{T_{T}}$.

In conclusion, a thermometer moving with four-velocity $v$ in a system in local thermodynamical equilibrium, characterized by a four-vector field $\beta$, will mark a temperature which is equal to

$T_{T}=\frac{1}{\beta(x) \cdot v}$.

As the scalar product of two timelike unit vectors $u \cdot v \geq 1$ and

$u \cdot v=1$ iff $u=v$

one has, according to (30),

$T_{T} \leq T_{0}=\frac{1}{\sqrt{\beta^{2}}} \quad T_{T}=T_{0} \quad$ iff $u=v$,

that is, the temperature marked by an idealized thermometer is maximal if it moves with the same four-velocity of the fluid. Thereby, we can establish a thought operational procedure to define a four-velocity of the fluid based on the notion of LTE at the spacetime point $x$ :
- put (infinitely many) ideal thermometers in contact with the relativistic system at the spacetime point $x$, each with a different four-velocity $v$;

- the ideal thermometer marking the highest value $T_{0}$ moves, by definition, with the four-velocity $u(x)=T_{0} \beta(x)=$ $1 / \sqrt{\beta^{2}}$.

\section{The stress-energy tensor in the $\beta$ frame}

As has been mentioned in Sect. 2, the LTE density operator that we have defined and discussed in Sect. 2 is not the true density operator $\widehat{\rho}$. In the Heisenberg representation, the true density operator is stationary, time-independent, which is evidently not the case for $\widehat{\rho}_{\mathrm{LE}}$ in Eq. (5), which depends on time $\tau$ so that the total entropy can change (in fact increase) in time. The true stationary density operator $\widehat{\rho}$ is the one needed to write the continuity equations of the mean values of operators, such as the stress-energy tensor:

$\partial_{\mu} T^{\mu \nu}=\partial_{\mu} \operatorname{tr}\left(\widehat{\rho} \widehat{T}^{\mu \nu}\right)_{\text {ren }}=\operatorname{tr}\left(\widehat{\rho} \partial_{\mu} \widehat{T}^{\mu \nu}\right)_{\text {ren }}=0$.

Equation (31) is the basic equation of relativistic hydrodynamics and, in the above form, makes it clear that the conservation of the mean value stems from the more fundamental conservation equation of the corresponding quantum operator.

If, at some initial time $\tau_{0}$, the system is known to be at local thermodynamical equilibrium, one can take the actual, timeindependent, density operator as the one in Eq. (5), provided that both the spacelike hypersurface $\Sigma$ and the operators $\widehat{T}$, $\widehat{j}$ are evaluated at $\tau_{0}$ :

$\widehat{\rho}=\frac{1}{Z} \exp \left[-\int_{\Sigma\left(\tau_{0}\right)} \mathrm{d} \Sigma n_{\mu}\left(\widehat{T}^{\mu v} \beta_{v}-\widehat{j}^{\mu} \xi\right)\right]$.

Consider now the evolution in $\tau$ of the LTE hypersurface $\Sigma$; one can then rewrite the density operator in (32) in terms of the operators at the present time $\tau$ by means of the Gauss' theorem:

$$
\begin{aligned}
\widehat{\rho}= & \frac{1}{Z} \exp \left[-\int_{\Sigma(\tau)} \mathrm{d} \Sigma n_{\mu}\left(\widehat{T}^{\mu v} \beta_{v}-\widehat{j}^{\mu} \xi\right)\right. \\
& \left.+\int_{\Omega} \mathrm{d} \Omega\left(\widehat{T}^{\mu v} d_{\mu} \beta_{v}-\widehat{j}^{\mu} d_{\mu} \xi\right)\right]
\end{aligned}
$$

where $d$ stands for the covariant derivative in the coordinates $\tau$ and $\sigma_{i} i=1,2,3$ of the surfaces $\Sigma$. The region $\Omega$ is the portion of spacetime enclosed by the two hypersurfaces $\Sigma\left(\tau_{0}\right)$ and $\Sigma(\tau)$ and the timelike hypersurface at their boundaries, where the flux of $\left(\widehat{T}^{\mu \nu} \beta_{v}(x)-\widehat{j}^{\mu} \xi(x)\right)$ is supposed to vanish (see e.g. Fig. 1).

The first term of the exponent on the right hand side of Eq. (33) is just the LTE exponent at time $\tau$. If the evolution of the stress-energy tensor and current operators are such that the system keeps close to a situation of local 
thermodynamical equilibrium - a requirement of relativistic hydrodynamics - the second term in the exponent can be considered as a perturbation with respect to the first term and, accordingly, an expansion can be made in the gradients of the $\beta$ and $\xi$ fields with the method of linear response theory, through an iterated use of the operator Kubo identity. This in essence, is the method put forward by Zubarev $[29,30]$ and used by Hosoya et al. [31] to generate so-called Green-Kubo formulas of transport coefficients for a relativistic fluid, which coincide with those obtained by using the method of the variation of the metric into the equilibrium euclidean action [1]. The expansion allows one to express the mean value of a spacetime-dependent operator $\widehat{O}(x)$ with $x=(\tau, \sigma)$ as the mean value at LTE plus a correction depending on the gradients:

$$
\begin{aligned}
\langle\widehat{O}(x)\rangle \simeq & \langle\widehat{O}(x)\rangle_{\mathrm{LE}}-\langle\widehat{O}(x)\rangle_{\mathrm{LE}}\langle\widehat{B}\rangle_{\mathrm{LE}} \\
& +\int_{0}^{1} \mathrm{~d} z\left\langle\widehat{O}\left(x^{\prime}\right) \mathrm{e}^{z \widehat{A}} \widehat{B} \mathrm{e}^{-z \widehat{A}}\right\rangle_{\mathrm{LE}}
\end{aligned}
$$

choosing the LTE hypersurface so as to go through the point $x$. In Eq. (34) the operators $\widehat{A}$ and $\widehat{B}$ are, respectively, the first and the second integral in the exponent of Eq. (33). In flat spacetime, the integration region $\Omega$ is bounded by the two LTE hypersurfaces at $\tau$ and $\tau_{0}$. They can be approximated by the spacelike tangent hyperplanes at the points $x=(\tau, \sigma)$ and $\left(\tau_{0}, \sigma\right)$ respectively, whose normal versor is $n_{\mu}$. This allows one to carry out the integration over Minkowski spacetime, with the time $t$ marked by an observer moving with velocity $n$, as well as replacing covariant derivative with usual derivatives:

$$
\begin{aligned}
& \int_{\Omega} \mathrm{d} \Omega\left(\widehat{T}^{\mu v} d_{\mu} \beta_{\nu}-\widehat{j}^{\mu} d_{\mu} \xi\right) \\
& \quad \rightarrow \int_{T \Omega} \mathrm{d}^{4} x\left(\widehat{T}^{\mu \nu} \partial_{\mu} \beta_{\nu}-\widehat{j}^{\mu} \partial_{\mu} \xi\right) .
\end{aligned}
$$

Altogether, this approach generates an expansion of the stress-energy tensor (as well as any operator) from the LTE point in the gradients of the thermodynamic fields $\beta$ and $\xi$ which is - as we will see-equivalent to that in the usual $u$, $T$, and $\mu$ :

$T^{\mu \nu}=\operatorname{tr}\left(\widehat{\rho} \widehat{T}^{\mu \nu}\right)_{\text {ren }}=T_{\mathrm{LE}}^{\mu v}(x)+\delta T^{\mu \nu}(\partial \beta, \partial \xi)$.

However, neither the hydrodynamical frame nor the zeroorder term of the expansion, that is, the mean value at LTE, were discussed in detail in Ref. [31], where it was simply assumed that $T_{\mathrm{LE}}^{\mu \nu}(x)$ has the familiar ideal form:

$T_{\mathrm{id}}^{\mu \nu}(x)=(\rho+p)_{\mathrm{eq}} \frac{1}{\beta^{2}} \beta^{\mu}(x) \beta^{\nu}(x)-p_{\mathrm{eq}} g^{\mu \nu}$.

In fact, as we will see, the zeroth-order term, that is,

$$
\begin{aligned}
& T_{\mathrm{LE}}^{\mu \nu}(x)=\operatorname{tr}\left(\widehat{\rho}_{\mathrm{LE}} \widehat{T}^{\mu \nu}(x)\right)_{\text {ren }}=\frac{1}{Z_{\mathrm{LE}}} \operatorname{tr} \\
& \left(\exp \left[-\int \mathrm{d} \Sigma_{\mu}\left(\widehat{T}^{\mu v} \beta_{\nu}-\xi \widehat{j}^{\mu}\right)\right] \widehat{T}^{\mu \nu}(x)\right)_{\text {ren }},
\end{aligned}
$$

is less trivial than generally believed and the choice of a hydrodynamical frame is crucial to determine its value. This is the subject of the remaining part of this section

\subsection{The stress-energy tensor at local thermodynamical equilibrium}

We first remark that, the $\beta$ being a function of the spacetime point, the trace in (37) cannot be calculated straightforwardly. However, in the exponent of $\widehat{\rho}_{\mathrm{LE}}$, one can make a Taylor expansion in $\beta$ and $\xi$ about the same point $x$ where the stressenergy tensor is to evaluated. The idea is that, at LTE, only the nearby points will contribute to its mean value, especially if the gradients are small. In other words, in the so-called hydrodynamical limit, the $\beta$ field is mostly uniform in the region where the stress-energy tensor correlation function, determined by microscopic correlation lengths, is significant. Hence

$$
\begin{aligned}
& \exp \left[-\int \mathrm{d} \Sigma_{\mu}\left(\widehat{T}^{\mu v} \beta_{v}-\xi \widehat{j}^{\mu}\right)\right] \\
& \simeq \exp \left[-\beta_{v}(x) \int \mathrm{d} \Sigma_{\mu} \widehat{T}^{\mu \nu}+\xi(x) \int \mathrm{d} \Sigma_{\mu} \widehat{j}^{\mu}-\frac{\partial \beta_{v}}{\partial \sigma_{i}}(x)\right. \\
& \quad \times \int \mathrm{d} \Sigma_{\mu} \widehat{T}^{\mu v}\left(\sigma_{i}-\sigma_{0 i}\right)+\frac{\partial \xi}{\partial \sigma_{i}}(x) \\
& \left.\quad \times \int \mathrm{d} \Sigma_{\mu} \widehat{j}^{\mu}\left(\sigma_{i}-\sigma_{0 i}\right)+\cdots\right] \\
& =\exp \left[-\beta_{v}(x) \widehat{P}^{v}+\xi(x) \widehat{Q}\right. \\
& \quad-\frac{\partial \beta_{v}}{\partial \sigma_{i}}(x) \int \mathrm{d} \Sigma_{\mu} \widehat{T}^{\mu \nu}\left(\sigma_{i}-\sigma_{0 i}\right) \\
& \left.\quad+\frac{\partial \xi}{\partial \sigma_{i}}(x) \int \mathrm{d} \Sigma_{\mu} \widehat{j}^{\mu}\left(\sigma_{i}-\sigma_{0 i}\right)+\ldots\right]
\end{aligned}
$$

where $\sigma$ are the curvilinear coordinates of the hypersurface $\Sigma$ at the time $\tau$ (the point $x$ has coordinates $\tau$ and $\sigma_{0}$ ). In the last equality we have taken into account that the integrals of the stress-energy tensor and the current over any $3 \mathrm{D}$ hypersurface equal the total four-momentum and charge. Now

$$
\begin{aligned}
\sum_{i=1}^{3} \frac{\partial \beta_{v}}{\partial \sigma_{i}}(x)\left(\sigma_{i}-\sigma_{0 i}\right) & =\sum_{i=1}^{3} \partial_{\lambda} \beta_{\nu}(x) \frac{\partial x^{\lambda}}{\partial \sigma_{i}}(x)\left(\sigma_{i}-\sigma_{0 i}\right) \\
& =\partial_{\lambda} \beta_{\nu}(x) \sum_{i=1}^{3} t_{i}^{\lambda}(x)\left(\sigma_{i}-\sigma_{0 i}\right),
\end{aligned}
$$

where $t_{\mu}^{i}$ are the vectors tangent to the hypersurface $\Sigma$. If the $\beta$ field is vorticity free, one can choose the $\beta$ frame with $n=\hat{\beta}$, thus the vectors $t^{i}$ will be simply orthogonal to $\beta$. Hence, denoting by $y$ the point with coordinates $\tau$ and $\sigma$, 


$$
\sum_{i=1}^{3} t_{i}^{\lambda}(x)\left(\sigma_{i}-\sigma_{0 i}\right) \simeq\left(y_{\lambda}-x_{\lambda}\right)_{T},
$$

where the subscript $T$ stands for the transverse projection with respect to $\beta$; introducing the definitions

$$
\begin{aligned}
& D \equiv u^{\mu} \partial_{\mu}=\frac{1}{\sqrt{\beta^{2}}} \beta^{\mu} \partial_{\mu}=T \beta^{\mu} \partial_{\mu} \\
& \nabla^{v} \equiv\left(g^{\mu \nu}-u^{\mu} u^{v}\right) \partial_{\mu}=\left(g^{\mu \nu}-T^{2} \beta^{\mu} \beta^{v}\right) \partial_{\mu} \equiv \Delta^{\mu \nu} \partial_{\mu}
\end{aligned}
$$

where $T=1 / \sqrt{\beta^{2}}$ is the comoving temperature, one can finally rewrite Eq. (38) as

$$
\begin{aligned}
& \exp \left[-\int \mathrm{d} \Sigma_{\mu}\left(\widehat{T}^{\mu v} \beta_{v}-\xi \widehat{j}^{\mu}\right)\right] \\
& \simeq \exp \left[-\beta_{v}(x) \widehat{P}^{v}+\xi(x) \widehat{Q}-\partial_{\lambda} \beta_{v}(x)\right. \\
& \times \int_{T \Sigma} \mathrm{d} \Sigma_{\mu}(y) \widehat{T}^{\mu \nu}(y)\left(y^{\lambda}-x^{\lambda}\right)_{T} \\
& \left.+\partial_{\lambda} \xi(x) \int_{T \Sigma} \mathrm{d} \Sigma_{\mu}(y) \widehat{j}^{\mu}(y)\left(y^{\lambda}-x^{\lambda}\right)_{T}+\cdots\right] \\
& =\exp \left[-\beta_{\nu}(x) \widehat{P}^{v}+\xi(x) \widehat{Q}-\nabla_{\lambda} \beta_{\nu}(x)\right. \\
& \int_{T \Sigma} \mathrm{d} \Sigma_{\mu}(y) \widehat{T}^{\mu v}(y)\left(y^{\lambda}-x^{\lambda}\right)_{T} \\
& \left.+\nabla_{\lambda} \xi(x) \int_{T \Sigma} \mathrm{d} \Sigma_{\mu}(y) \widehat{j}^{\mu}(y)\left(y^{\lambda}-x^{\lambda}\right)_{T}+\cdots\right] \\
& =\exp \left[-\beta_{\nu}(x) \widehat{P}^{v}+\xi(x) \widehat{Q}-\frac{1}{4}\left(\nabla_{\lambda} \beta_{\nu}(x)\right.\right. \\
& \left.-\nabla_{\nu} \beta_{\lambda}(x)\right) \widehat{J}_{x T}^{\lambda \nu}+\frac{1}{2}\left(\nabla_{\lambda} \beta_{\nu}(x)+\nabla_{\nu} \beta_{\lambda}(x)\right) \widehat{L}_{x}^{\lambda \nu} \\
& \left.+\nabla_{\lambda} \xi(x) \widehat{d}_{x}^{\lambda}+\cdots\right],
\end{aligned}
$$

where the integration - to a good approximation — can be carried out on the hyperplane $T \Sigma$ tangent to $\Sigma$ at the point $x$. In Eq. (40), the operator $\widehat{J}_{x T}$ is the transverse projection of the angular momentum operator around the point $x$ :

$\widehat{J}_{x T}^{\lambda \nu} \equiv \int_{T \Sigma} \mathrm{d} \Sigma(y) n_{\mu}\left(y^{\lambda}-x^{\lambda}\right)_{T} \widehat{T}^{\mu \nu}(y)-(\lambda \leftrightarrow \nu)$,

and we have

$$
\begin{aligned}
\widehat{L}_{x}^{\lambda v} & \equiv \frac{1}{2} \int_{T \Sigma} \mathrm{d} \Sigma(y) n_{\mu}\left(y^{\lambda}-x^{\lambda}\right)_{T} \widehat{T}^{\mu \nu}(y)+(\lambda \leftrightarrow v) \\
\widehat{d}_{x}^{\lambda} & \equiv \int_{T \Sigma} \mathrm{d} \Sigma(y) n_{\mu}\left(y^{\lambda}-x^{\lambda}\right)_{T} \widehat{j}^{\mu}(y) .
\end{aligned}
$$

We note in passing that $\widehat{L}_{x}$ and $\widehat{d}_{x}$ are not true tensors because they are integrals of non-conserved densities; their definition is only valid for the specific frame.

Unfortunately, the expression (40) does not imply the full correct global equilibrium limit (10). Particularly, it must be realized that this happens only if, at the global equilibrium defined by Eqs. (8) and (9) one has $\varpi_{\lambda \nu} \beta^{\lambda}=0$

However, in the global equilibrium with rotation (see Sect. 6), $\varpi_{\mu \nu} \beta^{\nu} \neq 0$ and it is proportional to the acceleration field. The reason for this shortcoming is the choice of $n=\hat{\beta}$, which is possible, as has been mentioned, only if the $\beta$ field is vorticity free, which is not true even for the simple case of rotating global equilibrium.

To find the correct expression it is convenient to use the decomposition in Eq. (24) to rewrite the integral in the exponent of LTE density operator (5) as

$$
\begin{aligned}
-\int \mathrm{d} \Sigma n_{\mu} \widehat{T}^{\mu \nu} \beta_{v}= & -\int \mathrm{d} \Sigma(y) n_{\mu} \widehat{T}^{\mu \nu}\left(b_{v}+\varpi_{\nu \lambda} y^{\lambda}\right) \\
= & -\int \mathrm{d} \Sigma(y) n_{\mu} \\
& \times\left[\widehat{T}^{\mu \nu} b_{v}-\frac{1}{2} \varpi_{\lambda \nu}\left(y^{\lambda} \widehat{T}^{\mu \nu}-y^{\nu} \widehat{T}^{\mu \lambda}\right)\right]
\end{aligned}
$$

where we have set $\xi=0$ for simplicity. We can now make a first-order Taylor expansion of the thermodynamic field $b$ in the integrand about the point $x$ and replace the integration domain with the hyperplane $T \Sigma$ tangent to $\Sigma$ in $x$ if necessary:

$$
\begin{aligned}
& -\int \mathrm{d} \Sigma(y) n_{\mu}\left[\widehat{T}^{\mu \nu} b_{v}-\frac{1}{2} \varpi_{\lambda \nu}\left(y^{\lambda} \widehat{T}^{\mu \nu}-y^{\nu} \widehat{T}^{\mu \lambda}\right)\right] \\
& \simeq-b_{\nu}(x) \int \mathrm{d} \Sigma(y) n_{\mu} \widehat{T}^{\mu \nu}-\frac{\partial b_{v}}{\partial x^{\rho}} \\
& \quad \times \int_{T \Sigma} \mathrm{d} \Sigma(y) n_{\mu}\left(y^{\rho}-x^{\rho}\right)_{T} \widehat{T}^{\mu \nu} \\
& \quad+\frac{1}{2} \int \mathrm{d} \Sigma(y) n_{\mu}\left(y^{\lambda} \widehat{T}^{\mu \nu}-y^{\nu} \widehat{T}^{\mu \lambda}\right) \varpi_{\lambda \nu}(y)
\end{aligned}
$$

where the subscript $T$ now stands for orthogonal to the vector $b(x)$. The integral in the first term on the right hand side of the above equation is just the four-momentum $\widehat{P}^{v}$, while the second term can be decomposed into symmetric and antisymmetric contributions. Since the $b$ field has a vanishing antisymmetric gradient [see Eq. (23)], one is left with

$$
\begin{aligned}
& -b_{\nu}(x) \widehat{P}^{\nu}-\frac{1}{4}\left(\partial_{\rho} b_{\nu}+\partial_{\nu} b_{\rho}\right) \int_{T \Sigma} \mathrm{d} \Sigma(y) n_{\mu} \\
& \quad \times\left[\left(y^{\rho}-x^{\rho}\right)_{T} \widehat{T}^{\mu \nu}+\left(y^{\nu}-x^{\nu}\right)_{T} \widehat{T}^{\mu \rho}\right] \\
& +\frac{1}{2} \int \mathrm{d} \Sigma(y) n_{\mu}\left(y^{\lambda} \widehat{T}^{\mu \nu}-y^{\nu} \widehat{T}^{\mu \lambda}\right) \varpi_{\lambda \nu}(y)
\end{aligned}
$$

where we have used the orthogonality between the tangent vectors to $\Sigma$ and $b$ implied by the choice $n=\hat{\beta}$ and the fact that $b$ field has vanishing external derivative [see Eq. (23)]; thus, only the symmetric combination of integral and derivatives of $b$ in Eq. (42) is retained.

We now want to work out and further expand (43) so as to have in it only linear terms in the first-order $\beta$ derivatives. As a first step, we can Taylor expand the tensor $\varpi$ in the last integral expression in Eq. (43) about the same point $x$ as for 
$b$; because of (25), in this expansion we will only retain the zeroth-order term if second-order derivatives of $\beta$ are not to appear and approximate $\varpi(x)$ with the antisymmetric part of the $\beta$ gradient in $x$. Secondly, we note that, according to the definition (24) and Eq. (25), the symmetric part of the gradient of $b$ differs from the corresponding symmetric part of the gradient of $\beta$ by terms involving higher-order derivatives:

$\partial_{\rho} b_{v}+\partial_{\nu} b_{\rho}=\partial_{\rho} \beta_{v}+\partial_{v} \beta_{\rho}+\mathcal{O}\left(\partial^{2}\right)$.

Therefore, we can rewrite Eq. (43) as

$$
\begin{aligned}
& -b_{\nu}(x) \widehat{P}^{\nu}-\frac{1}{4}\left(\partial_{\rho} \beta_{\nu}+\partial_{\nu} \beta_{\rho}\right) \int_{T \Sigma} \mathrm{d} \Sigma(y) n_{\mu} \\
& \quad \times\left[\left(y^{\rho}-x^{\rho}\right)_{T} \widehat{T}^{\mu \nu}+\left(y^{\nu}-x^{\nu}\right)_{T} \widehat{T}^{\mu \rho}\right] \\
& \quad+\frac{1}{2} \varpi_{\lambda \nu}(x) \int \mathrm{d} \Sigma(y) n_{\mu}\left(y^{\lambda} \widehat{T}^{\mu \nu}-y^{\nu} \widehat{T}^{\mu \lambda}\right) \\
& =-b_{v}(x) \widehat{P}^{\nu}-\frac{1}{4}\left(\partial_{\rho} \beta_{\nu}+\partial_{\nu} \beta_{\rho}\right) \\
& \quad \times \int_{T \Sigma} \mathrm{d} \Sigma(y) n_{\mu}\left[\left(y^{\rho}-x^{\rho}\right)_{T} \widehat{T}^{\mu \nu}\right. \\
& \left.+\left(y^{\nu}-x^{\nu}\right)_{T} \widehat{T}^{\mu \rho}\right]+\frac{1}{2} \varpi_{\lambda v}(x) \widehat{J}^{\lambda \nu} .
\end{aligned}
$$

The integrand argument $\left(y^{\rho}-x^{\rho}\right)_{T}$ is transverse to $b$ in the point $x$, which means that it is also transverse to $\beta$ up to first-order derivatives of $\beta$ in view of Eq. (8). Hence

$$
\begin{aligned}
\Delta_{b}^{\mu \nu}(x)= & g^{\mu \nu}-\frac{b^{\mu}(x) b^{\nu}(x)}{b^{2}(x)} \simeq g^{\mu \nu} \\
& -\frac{\beta^{\mu}(x) \beta^{\nu}(x)}{\beta^{2}(x)}+\mathcal{O}(\partial \beta) \simeq \Delta_{\beta}^{\mu \nu}(x) .
\end{aligned}
$$

We can then replace the transverse projector on the hypersurface orthogonal to $b$ with the one transverse to $\beta$ in Eq. (41) and write

$$
-b_{v}(x) \widehat{P}^{v}+\frac{1}{2} \varpi_{\lambda v}(x) \widehat{J}^{\lambda v}-\frac{1}{2}\left(\partial_{\lambda} \beta_{v}+\partial_{\nu} \beta_{\lambda}\right) \widehat{L}_{x}^{\lambda v}
$$

where $\widehat{L}_{x}$ is defined in Eq. (41). Finally, by using the identity (11) and the relation (24), one can rewrite Eq. (44) as

$$
-\beta_{\nu}(x) \widehat{P}^{v}+\frac{1}{2} \varpi_{\lambda v}(x) \widehat{J}_{x}^{\lambda v}-\frac{1}{2}\left(\partial_{\lambda} \beta_{\nu}+\partial_{\nu} \beta_{\lambda}\right) \widehat{L}_{x}^{\lambda \nu},
$$

and, finally, restoring the chemical potential term and replacing $\varpi$ with its first-order approximation in the $\beta$ derivatives,

$$
\begin{aligned}
& \widehat{\rho}_{\mathrm{LE}} \simeq \frac{1}{Z_{\mathrm{LE}}} \exp \left[-\beta_{\nu}(x) \widehat{P}^{v}+\xi(x) \widehat{Q}-\frac{1}{4}\left(\partial_{\nu} \beta_{\lambda}(x)\right.\right. \\
& \left.\left.-\partial_{\lambda} \beta_{\nu}(x)\right) \widehat{J}_{x}^{\lambda v}+\frac{1}{2}\left(\partial_{\nu} \beta_{\lambda}(x)+\partial_{\lambda} \beta_{\nu}(x)\right) \widehat{L}_{x}^{\lambda v}+\nabla_{\lambda} \xi(x) \widehat{d}_{x}^{\lambda}\right] .
\end{aligned}
$$

It can be seen that this expression has the correct global equilibrium limit in Eq. (8): as has been mentioned, the coefficient of $\widehat{L}$ and $\widehat{d}$ vanish because of Eq. (7) and $\varpi=$ const is given by the external derivative of the $\beta$ field like in Eq. (25).

The expression (46), once (25) is taken into account, implies that $\widehat{\rho}_{\text {LE }}$ can again be expanded in the gradients of the $\beta$ and $\xi$ fields with linear response theory starting from a point of global thermodynamical equilibrium with constant inverse four-temperature $\beta(x)$ and chemical potential $\xi(x) T(x)$, where

$\widehat{\rho}_{\mathrm{eq}}=\frac{1}{Z_{\mathrm{eq}}} \operatorname{tr}\left(\exp \left[-\beta_{v}(x) \widehat{P}^{v}+\xi(x) \widehat{Q}\right]\right)$.

Therefore

$$
\begin{aligned}
T_{\mathrm{LE}}^{\mu \nu}(x) \simeq & \frac{1}{Z_{\mathrm{eq}}(\beta(x), \xi(x))} \operatorname{tr}\left(\operatorname { e x p } \left[-\beta_{\nu}(x) \widehat{P}^{\nu}\right.\right. \\
& \left.+\xi(x) \widehat{Q}] \widehat{T}^{\mu \nu}(x)\right)_{\mathrm{ren}}+\mathcal{O}(\partial \beta, \partial \xi) .
\end{aligned}
$$

The first term of the expansion can be readily identified: it is the mean value of the stress-energy tensor at the global thermodynamical equilibrium with a global inverse temperature four-vector and a chemical potential equal to those in $x$. In other words, it is the ideal part of the stress-energy tensor, and the above expansion can be written as

$$
\begin{aligned}
T_{\mathrm{LE}}^{\mu \nu}(x) \simeq & T_{\mathrm{id}}^{\mu \nu}(x)+\mathcal{O}\left((\partial \beta, \partial \xi)^{N}\right) \\
= & (\rho+p)_{\mathrm{eq}} \frac{1}{\beta^{2}} \beta^{\mu}(x) \beta^{\nu}(x)-p_{\mathrm{eq}} g^{\mu \nu} \\
& +\mathcal{O}\left((\partial \beta, \partial \xi)^{N}\right),
\end{aligned}
$$

where the energy density $\rho$ and pressure $p$ are the same thermodynamic functions of $\beta^{2}(x), \xi(x)$ as at equilibrium. Equation (47) shows that the mean value of the stress-energy tensor differs from the ideal one by terms which, potentially, are of the first order in the gradients of $\beta$ and $\xi$.

We believe, though we do not present any calculation here, that first-order terms in the gradient expansion of the mean value at LTE are vanishing, owing to general symmetry requirements. Instead, second-order terms in the expansion of the operator (46) should be non-vanishing, whence $N=2$ in (47). Some of the coefficients in the second-order gradient expansion have been recently calculated in Ref. [32]. Therefore, our terms obtained from an expansion of the LTE expression would either coincide with them-specifically the non-dissipative which survive, e.g., in the global equilibrium rotating case proportional to $\varpi \varpi$-or additionally contribute to the second-order dissipative coefficients, specifically those proportional to $\sigma \sigma$ or $\sigma \varpi$ where $\sigma$ is the symmetric part of $\partial_{\mu} \beta_{\nu}$. This will be the subject of further work.

\section{The $\beta$ frame vs. Landau frame}

We now come to a major point, namely the discussion of the difference between the $\beta$ frame and the familiar Landau 
frame. In the previous section we have seen that, in the $\beta$ frame, Eq. (35) holds, and an equivalent one also holds for $j$ :

$T^{\mu \nu}=T_{\mathrm{LE}}^{\mu \nu}+\delta T^{\mu \nu} \quad j^{\mu}=j_{\mathrm{LE}}^{\mu}+\delta j^{\mu}$.

If the $\beta$ field is non-vortex, then, because of Eq. (15),

$\beta_{\mu} \delta T^{\mu \nu}=0 \quad \beta_{\mu} \delta j^{\mu}=0$.

Indeed, the first of the two equations (48) apparently imposes the orthogonality between the viscous part of the stressenergy tensor and the velocity vector, a condition often referred to as "Landau matching condition", so naively one would say that the $\beta$ frame and the Landau frame are equivalent, at least as long as $\beta$ is vorticity free. However, the actual definition of the Landau frame prescribes that the velocity four-vector $u_{L}$ is the timelike eigenvector of $T$

$T^{\mu v} u_{L v}=\lambda u_{L}^{\mu}$.

It is worth remarking that the above Landau frame definition provides four independent equations, whereas the definition of the $\beta$ frame involves five equations. In fact, the Landau frame definition is usually, often tacitly, supplemented by the equality of the proper charge density, respectively, with its local equilibrium value

$u_{L} \cdot j=u_{L} \cdot j_{\mathrm{LE}}$,

which indeed amounts to enforce the second equality in the Eq. (48). In the traditional Landau scheme, this equation is sometimes justified through a redefinition of the temperature and chemical potential [33] in a non-equilibrium situation. However, as we have emphasized in this work, temperature and chemical potential can be unambiguously defined at the LTE; see Sect. 2. In fact, when changing frames, it should always be checked whether the basic relations involving thermodynamical quantities hold with the accordingly defined temperature and chemical potential.

Equation (48) implies Eq. (49) only if $\beta$ is an eigenvector of $T_{\mathrm{LE}}^{\mu \nu}$

$0=\beta_{\mu} \delta T^{\mu \nu}=\beta_{\mu}\left(T^{\mu \nu}-T_{\mathrm{LE}}^{\mu \nu}\right)=\beta_{\mu} T^{\mu \nu}-\lambda \beta^{\nu}$,

whence $\beta$ is the timelike eigenvector of $T$, so $\hat{\beta} \equiv u_{L}$.

So, the $\beta$ frame coincides with the Landau frame if $\beta$ is vorticity free and if it is the timelike eigenvector of $T_{\mathrm{LE}}$. In all other cases, including the case of a vortex $\beta$ field, the Landau and $\beta$ frame are not equivalent.

It can be readily realized that $\beta$ is an eigenvector of $T_{\mathrm{LE}}$ if $T_{\mathrm{LE}}=T_{\mathrm{id}}$. However, we have seen at the end of Sect. 5 that this is not generally the case for the quantum form of LTE, i.e. there may be corrections to the ideal stress-energy tensor depending on the gradients of the $\beta$ field itself whose leading terms are expected to be quadratic.

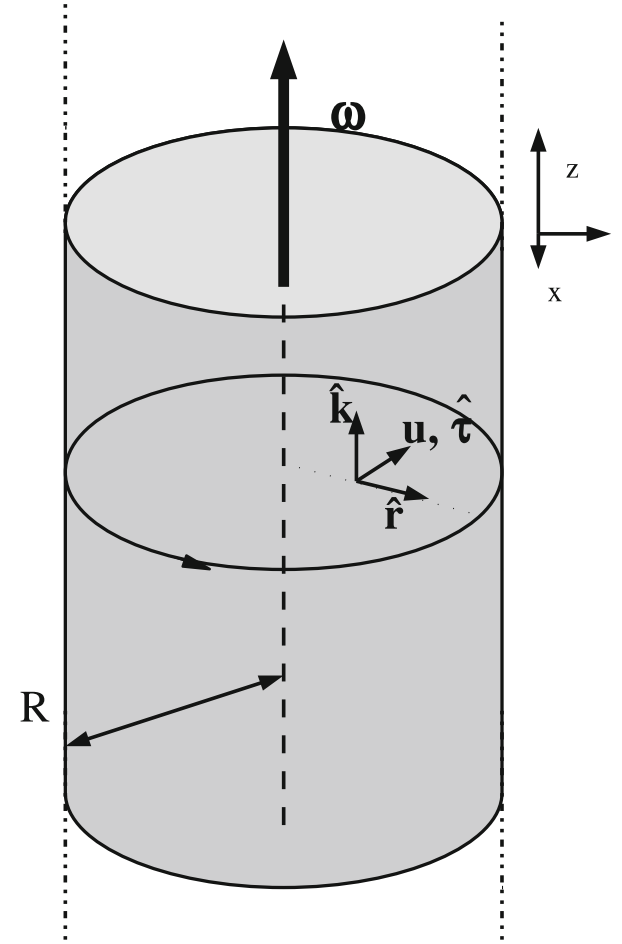

Fig. 2 Rotating cylinder with finite radius $R$ at temperature $T$. Also shown the inertial frame axes and the spatial parts of the vectors of tetrad

We are now going to discuss in detail a remarkable instance of inequivalence between Landau and $\beta$ frames: the rotational ensemble, which is a global equilibrium case. Its density operator can be obtained from Eq. (10) by setting

$b=\left(1 / T_{0}, 0,0,0\right) \varpi_{\mu \nu}=\left(\omega / T_{0}\right)\left(g_{1 \mu} g_{2 \nu}-g_{1 \nu} g_{2 \mu}\right)$,

that is,

$\widehat{\rho}=\frac{1}{Z} \exp \left[-\widehat{H} / T_{0}+\omega \widehat{J}_{z} / T_{0}\right] \mathrm{P}_{V}$,

where $\widehat{J}_{z}$ is the angular momentum operator along some fixed axis $z$ and $\omega$ has the physical meaning of a constant angular velocity (see Fig. 2); $P_{V}$ is a projector onto localized states, those obtained by enforcing peculiar boundary conditions on the quantum fields at some radius $R$ of an indefinitely long cylinder with axis $z$ and such that $\omega R<c$ (see [26]). With the above choice of $b$ and $\varpi$, the relevant $\beta$ field in Eq. (8) reads

$\beta=\frac{1}{T_{0}}(1, \omega \times \mathbf{x})$

where $\omega=\omega \hat{\mathbf{k}}$. Its field lines are then circles centered on the $z$ axis (see Fig. 2). Note that $1 / \sqrt{\beta^{2}} \equiv T \neq T_{0}$, that is, the proper temperature differs from the constant "global" temperature $T_{0}$, a well-known relativistic feature. The density operator (51) is independent of the spacelike hypersurface $\Sigma$ 
(i.e. time-independent) provided that the fluxes of $\widehat{T}^{\mu v} \beta_{v}$ and $\widehat{j}^{\mu}$ vanish at the boundary,

$$
\int_{\text {Boundary }} \mathrm{d} \Sigma_{\mu}\left(\widehat{T}^{\mu v} \beta_{v}-\xi \widehat{j}^{\mu}\right)=0 \text {. }
$$

In the usual formulation of relativistic hydrodynamics in the Landau frame the stress-energy tensor is decomposed along the $u_{L}$ vector as follows:

$T^{\mu \nu}=\left(\rho_{L}+p\right) u_{L}^{\mu} u_{L}^{\nu}-p g^{\mu \nu}+\Pi^{\mu \nu}$,

with $\Pi^{\mu v} u_{L v}=0$ by definition of $u_{L}$ but with the understood assumption that $\Pi \rightarrow 0$ at LTE [34] and, a fortiori, at the global thermodynamical equilibrium. We will show that in the rotational case one has $\hat{\beta} \neq u_{L}$ as well as $\Pi \neq 0$.

The latter inequality is expected to be a consequence of the fact that the density operator (51) has a cylindrical symmetry along $z$ axis, but not a full rotational symmetry, so there is no principal reason why the mean stress-energy tensor ought to be isotropic in its local (Landau) rest frame, or, in other words, why its spacelike eigenvalues ought to be the same. Indeed, with cylindrical symmetry, its most general form reads

$$
\begin{aligned}
T^{\mu \nu}= & G(r) u^{\mu} u^{\nu}+H(r)\left(\hat{\tau}^{\mu} u^{\nu}+\hat{\tau}^{\nu} u^{\mu}\right) \\
& +I(r)\left(\hat{r}^{\mu} u^{\nu}+\hat{r}^{\nu} u^{\mu}\right)+J(r) \hat{\tau}^{\mu} \hat{\tau}^{\nu} \\
& +K(r)\left(\hat{r}^{\mu} \hat{\tau}^{\nu}+\hat{r}^{\nu} \hat{\tau}^{\mu}\right)+L(r) \hat{r}^{\mu} \hat{r}^{\nu}-M(r) g^{\mu \nu},
\end{aligned}
$$

where $G, H, I, J, K, L, M$ are generic functions of the radial coordinate $r$ such that $H(0)=I(0)=K(0)=0, u=$ $\hat{\beta}, \hat{r}=(0, \hat{\mathbf{r}}), \hat{k}=(0, \hat{\mathbf{k}})$, and $\hat{\tau}$ is the spacelike versor orthogonal to the previous three, that is,

$\hat{\tau}=(\gamma v, \gamma \hat{\mathbf{v}})$

where $\mathbf{v}=\omega \times \mathbf{x}$ and $\gamma=\left(1-v^{2}\right)^{-1 / 2}$ (see Fig. 2). The condition $\partial_{\mu} T^{\mu \nu}=0$ allows one to eliminate either $I(r)$ or $K(r)$ and this entails some differential relations between the functions in Eq. (54). Clearly, if in Eq. (54) either $H$ or $I$ or both are non-vanishing, the four-vector $u=\hat{\beta}$ is not an eigenvector of $T$ and the Landau and $\beta$ frame differ. Furthermore, if the scalar functions in (54) do not meet specific relations, the diagonal form of the tensor at equilibrium is not the ideal one (no isotropy) and the understood assumption $\Pi=0$ at equilibrium for the decomposition (53) breaks down. The rotational ensemble gives an opportunity to discuss in more detail the relevance of these effects, namely the magnitude of the difference between $\hat{\beta}$ and $u_{L}$ and the relevant scales. As we pointed out at the end of Sect. 5, the leading global equilibrium corrections to the stress-energy tensor are quadratic in the tensor $\varpi$, that is, the antisymmetric part of $\partial \beta$. In natural units this tensor is non-dimensional and its magnitude in the rotational case, as implied by Eq. (50), is $\hbar \omega / K T_{0}$ (natural constants are purposely restored here). This means that the deviation from the familiar hydrodynamical scheme scales like $\left(\hbar \omega / K T_{0}\right)^{2}$, which is a tiny number in most cases; still it can become relevant in special circumstances. It is worth stressing that $\hbar \omega / K T_{0}$, tiny as it might be, is a further macroscopic scale independent of the microscopic scales such as a correlation length or a thermal wavelength. We can otherwise say that the stress-energy tensor deviations from the ideal form at equilibrium are to be expected in the presence of a local acceleration such as when the velocity field is rigid. This will be the subject of further work.

As an example we calculate the stress-energy tensor of the free scalar real field (for details see Appendix A). The boundary condition at the outer surface $r=R$ of the cylinder is $\widehat{\psi}(R)=0$, which ensures the necessary vanishing of the flux Eq. (52). Indeed, since $\widehat{\psi}(R)=0$, the gradient of the field at $r=R$ is normal to the outer surface, that is,

$\left.\partial_{\mu} \widehat{\psi}\right|_{r=R}=\widehat{\chi}(t, R, \phi, z) \hat{r}^{\mu}$.

Since for the free scalar field ${ }^{3}$

$\widehat{T}^{\mu \nu}=\partial^{(\mu} \widehat{\psi} \partial^{\nu)} \widehat{\psi}-g^{\mu \nu} \widehat{\mathcal{L}} \widehat{\mathcal{L}}=\frac{1}{2}\left(\partial_{\mu} \widehat{\psi} \partial^{\mu} \widehat{\psi}-m^{2} \widehat{\psi}^{2}\right)$,

one has

$\hat{r}_{\mu} \widehat{T}^{\mu v}(R) \beta_{v}=0 ;$

hence Eq. (52), taking into account that $\xi=0$. Furthermore, the condition (56) make, at the operator level, the fluxes of the energy and angular momentum outside the cylinder boundary vanishing, implying the conservation of $\widehat{H}$ and $\widehat{J}_{z}$, as it should be.

Unlike for the ideal case (36), $T^{\mu v} \beta_{\nu}$ is not parallel to $\beta^{\mu}$. Indeed, one has

$$
\begin{aligned}
\hat{\tau}_{\mu} T^{\mu v} \beta_{v}= & \sqrt{\beta^{2}} 2 \gamma^{2} \sum_{M=-\infty}^{+\infty} \sum_{p_{T}} \\
& \times \int \mathrm{d} p_{L} \frac{J_{M}^{2}\left(p_{T} r\right)}{(2 \pi)^{2} \varepsilon R^{2} J_{M}^{\prime 2}\left(p_{T} R\right)} \frac{1}{\mathrm{e}^{(\varepsilon-M \omega) / T_{0}-1}} \\
& \times\left[\omega r\left(\varepsilon^{2}+\frac{M^{2}}{r^{2}}\right)-\left(1+\omega^{2} r^{2}\right) \frac{\varepsilon M}{r}\right]
\end{aligned}
$$

where $p_{T}$ are the discrete values related to the zeros $\zeta_{l, M}$ of the Bessel function $J_{M}$ by $p_{T} R=\zeta_{l, M}$ and $\varepsilon=$ $\sqrt{p_{T}^{2}+p_{L}^{2}+m^{2}}$; see Appendix A. As expected, the above expression is vanishing for $\omega=0$, i.e. in the non-rotating case, but for $\omega \neq 0$ it is non-vanishing, which is confirmed by numerical computation, shown in Fig. 3. This computation was carried out in the non-relativistic limit with $m \gg T_{0}$ and for $r$ values such that $\omega r \ll 1$. Still, the covered range in $r$ far exceeds the typical microscopic length, that is, the thermal

\footnotetext{
3 The round brackets on indices stand for symmetrization.
} 

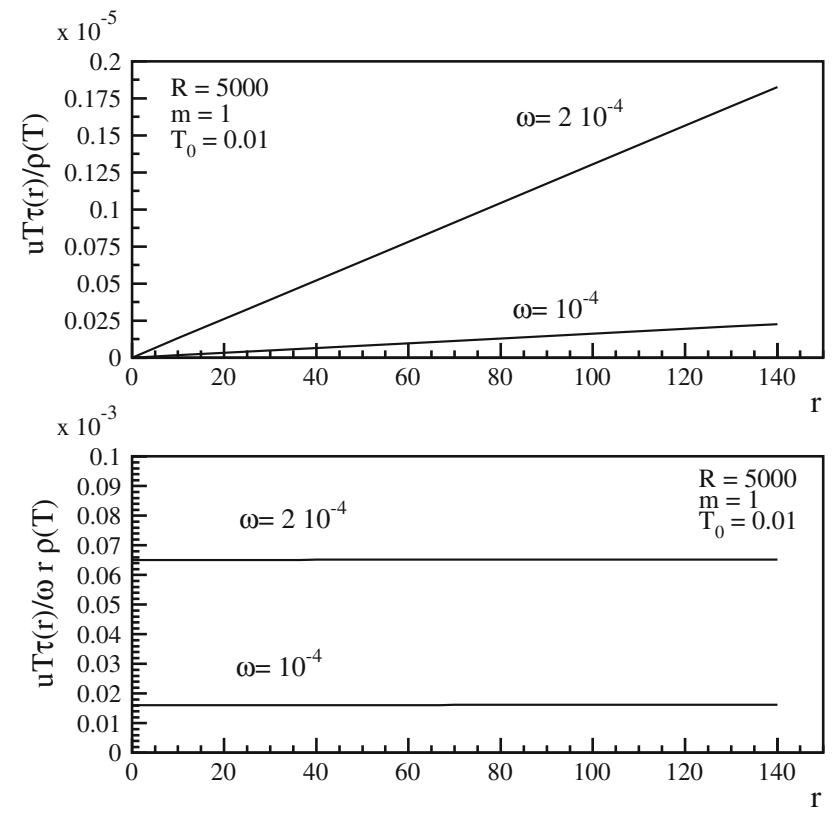

Fig. 3 Ratio between the projection $u_{\mu} \hat{\tau}_{v} T^{\mu \nu}$ for the free scalar field at global thermodynamical equilibrium within a rotating cylinder and the usual energy density of an ideal scalar gas as a function of the radial distance $r$. The radius $R$ is in arbitrary units and the values of $\omega, T_{0}, R$ and $m$ lie in the non-relativistic domain

wavelength scale $1 / \sqrt{m T_{0}}$, which turns out to be 0.1 in the distance units of Fig. 3. It can be seen that the ratio between the stress-energy tensor component $\tau \cdot T \cdot u$ in Eq. (58) and the usual energy density expression of an ideal massive scalar is non-vanishing. It increases linearly as a function of $r$, which is owing to the fact that $\tau \cdot T \cdot u \simeq \tau^{0} u^{0} T^{00}$ in the non-relativistic limit and that $\tau^{0} \simeq \omega r$ [see definition (55)]. Once $\tau \cdot T \cdot u$ is divided by $\omega r$, it can be seen that the ratio between this component and the usual energy density is approximately constant for small $r$. Moreover, its value is of the order $\left(\omega / T_{0}\right)^{2}=10^{-4}$ with an exact scaling with $\omega^{2}$, as expected. This demonstrates the difference between $\beta$ and Landau frame.

The full stress-energy tensor turns out to be

$$
T=\left(\begin{array}{cccc}
u \cdot T \cdot u & u \cdot T \cdot \hat{\tau} & 0 & 0 \\
u \cdot T \cdot \hat{\tau} & \hat{\tau} \cdot T \cdot \hat{\tau} & 0 & 0 \\
0 & 0 & \hat{r} \cdot T \cdot \hat{r} & 0 \\
0 & 0 & 0 & k \cdot T \cdot k
\end{array}\right)
$$

where the quoted components are non-vanishing and calculated in Appendix A. In conclusion, $\hat{\beta}$ is not the timelike eigenvector $u_{L}$ of $T$ and the Landau and the $\beta$ frame are not equivalent in this case. While it is possible to express $u_{L}$ as a superposition of $u=\hat{\beta}$ and $\tau$, the two spacelike eigenvectors $n$ and $k$ have different eigenvalues, because (see again Appendix A)

$$
\begin{aligned}
\hat{r} \cdot T \cdot \hat{r}-k \cdot T \cdot k \\
=\sum_{M=-\infty}^{+\infty} \sum_{p_{T}} \int \mathrm{d} p_{L} \frac{2}{(2 \pi)^{2} \varepsilon R^{2} J_{M}^{\prime 2}\left(p_{T} R\right)} \\
\quad \times \frac{1}{\mathrm{e}^{(\varepsilon-M \omega) / T_{0}}-1}\left[p_{T}^{2} J_{M}^{\prime}\left(p_{T} r\right)^{2}-p_{L}^{2} J_{M}\left(p_{T} r\right)^{2}\right],
\end{aligned}
$$

which is not vanishing. This can be readily checked by setting $r=R$ and using the boundary condition of the Bessel function. Consequently, at thermodynamical equilibrium, the term $\Pi$ in the decomposition (53) is non-vanishing, unlike commonly assumed. Another important consequence of the difference between $\beta$ and Landau frame is that the basic local thermodynamic relation (20) cannot be the same in both frames (see Appendix B).

\section{Equations of relativistic hydrodynamics in the $\beta$ frame}

The $\beta$ frame is an especially suitable framework to write the equations of relativistic hydrodynamics. As is well known, the general problem is to determine the evolution of the stress-energy tensor, and possibly several vector currents starting from definite initial conditions, under the assumption of approximate local thermodynamical equilibrium. This condition, in the case of one conserved current, as we have seen, reduces the number of unknown functions to five, that is, the four components of $\beta$ and $\xi$, which is just the number of continuity equations. In terms of these variables, the equations of relativistic hydrodynamics do not show any distinction between equations of motion and equation of state (which is encoded in the dependence of the pressure on $\beta^{2}$ and $\xi$, as we will see).

\subsection{Ideal hydrodynamics}

As we have seen in Sect. 5 the stress-energy tensor in $x$ at the lowest order in the gradient expansion can be approximated by the ideal one $T_{\mathrm{id}}^{\mu \nu}$ with inverse temperature four-vector and the chemical potential equal to those in the point $x$. We have shown in Ref. [22] that it can be obtained by taking derivatives of the thermodynamic potential current $\phi^{\mu}=p \beta^{\mu}$, where $p$ is the equilibrium pressure, a scalar field depending on the scalars $\beta^{2}$ and $\xi$. Thus

$T_{\mathrm{id}}^{\mu \nu}=-2 \frac{\partial p}{\partial \beta^{2}} \beta^{\mu} \beta^{\nu}-p g^{\mu \nu}$,

the derivative of the pressure being proportional to the proper enthalpy density,

$-2 \frac{\partial p}{\partial \beta^{2}}=\frac{\rho+p}{\beta^{2}}=\frac{h}{\beta^{2}}$. 
Similarly

$j_{\mathrm{eq}}^{\mu}=\frac{\partial p}{\partial \xi} \beta^{\mu}$,

the derivative of the pressure with respect to $\xi$ being proportional to the charge density

$\frac{\partial p}{\partial \xi}=\frac{q}{\sqrt{\beta^{2}}}$.

These expressions allow one to reformulate ideal relativistic hydrodynamics through five unknown functions: the fourvector $\beta$ (whose modulus is the inverse local temperature) and the scalar field $\xi$, corresponding to the ratio $\mu / T$. There are indeed five differential equations corresponding to the conservation equations of $T$ and $j$, which in principle allow one to solve the problem, provided that the functional relation $p\left(\beta^{2}, \xi\right)$ is known, which is but the complete equation of state. Nothing new, however; the introduction of these variables as primary fields allows one to gain further insight into the structure and features of relativistic hydrodynamics.

At the lowest order in the gradients, using Eq. (35), the continuity equations are those of the ideal hydrodynamics,

$$
\begin{aligned}
\partial_{\mu} T_{\mathrm{id}}^{\mu \nu}= & -2 \frac{\partial^{2} p}{\partial \beta^{22}} \beta^{\mu} \beta^{\nu} \partial_{\mu} \beta^{2}-2 \frac{\partial p}{\partial \beta^{2} \partial \xi} \beta^{\nu} \beta^{\mu} \partial_{\mu} \xi \\
& -2 \frac{\partial p}{\partial \beta^{2}}\left(\beta^{\nu} \partial \cdot \beta+\beta^{\mu} \partial_{\mu} \beta^{\nu}\right)-\frac{\partial p}{\partial \beta^{2}} \partial^{\nu} \beta^{2} \\
& -\frac{\partial p}{\partial \xi} \partial^{v} \xi=\mathcal{O}\left(\partial^{2}\right) \simeq 0
\end{aligned}
$$

and

$$
\begin{aligned}
\partial_{\mu} j_{\mathrm{eq}}^{\mu}= & \frac{\partial^{2} p}{\partial \xi^{2}} \beta^{\mu} \partial_{\mu} \xi+\frac{\partial^{2} p}{\partial \beta^{2} \partial \xi} \beta^{\mu} \partial_{\mu} \beta^{2} \\
& +\frac{\partial p}{\partial \xi} \partial \cdot \beta=\mathcal{O}\left(\partial^{2}\right) \simeq 0 .
\end{aligned}
$$

Since

$\partial \cdot \beta=\frac{D \beta^{2}}{2 \sqrt{\beta^{2}}}+\nabla \cdot \beta$,

Eq. (62) can be written, at the lowest order, as

$\frac{\partial^{2} p}{\partial \xi^{2}} \sqrt{\beta^{2}} D \xi+\frac{\partial^{2} p}{\partial \beta^{2} \partial \xi} \sqrt{\beta^{2}} D \beta^{2}+\frac{\partial p}{\partial \xi} \partial \cdot \beta=0$,

and Eq. (61), at the lowest order, can be split into two equations projecting along $\beta$ and transversely to it using (39),

$$
\begin{gathered}
\frac{\partial^{2} p}{\partial \beta^{22}} \beta^{2} \sqrt{\beta^{2}} D \beta^{2}+\frac{\partial^{2} p}{\partial \beta^{2} \partial \xi} \beta^{2} \sqrt{\beta^{2}} D \xi+\frac{\partial p}{\partial \beta^{2}} \beta^{2} \\
\times\left(\frac{3}{2 \sqrt{\beta^{2}}} D \beta^{2}+\nabla \cdot \beta\right)+\frac{1}{2} \frac{\partial p}{\partial \xi} \sqrt{\beta^{2}} D \xi=0 \\
\frac{\partial p}{\partial \beta^{2}}\left(\sqrt{\beta^{2}} \Delta_{\mu \nu} D \beta^{\nu}+\frac{1}{2} \nabla_{\mu} \beta^{2}\right)+\frac{1}{2} \frac{\partial p}{\partial \xi} \nabla_{\mu} \xi=0 .
\end{gathered}
$$

These two equations are the relativistic generalizations of the continuity equations and the Euler equation of motion of the fluid. We can readily retrieve its familiar form by noting that $\frac{1}{2} \frac{\partial p}{\partial \beta^{2}} \nabla_{\mu} \beta^{2}+\frac{1}{2} \frac{\partial p}{\partial \xi} \nabla_{\mu} \xi=\frac{1}{2} \nabla_{\mu} p$

and

$\sqrt{\beta^{2}} \Delta_{\mu \nu} D \beta^{\nu}=\sqrt{\beta^{2}} \Delta_{\mu \nu} D\left(\frac{1}{T} u^{\nu}\right)=\Delta_{\mu \nu} \beta^{2} D u^{\nu}=\beta^{2} A_{\mu}$,

$A^{v}=D u^{v}$ being the acceleration by definition. The second of (64) then becomes, by using (60),

$$
\frac{\partial p}{\partial \beta^{2}} \beta^{2} A_{\mu}=-\frac{1}{2}(\rho+p) A_{\mu}=-\frac{1}{2} \nabla_{\mu} p,
$$

that is, the well-known form of the relativistic Euler equation.

It is interesting to note that the first term in the relativistic Euler equation in (64) can also be written as

$\sqrt{\beta^{2}} \Delta_{\mu \nu} D \beta^{\nu}+\frac{1}{2} \nabla_{\mu} \beta^{2}=\beta_{\lambda} \Delta_{\mu \nu}\left(\partial^{\lambda} \beta^{\nu}+\partial^{\nu} \beta^{\lambda}\right)$

as well as

$$
\begin{aligned}
\sqrt{\beta^{2} \Delta_{\mu \nu} D \beta^{\nu}+\frac{1}{2} \nabla_{\mu} \beta^{2}} & =\beta^{2} A^{\mu}+\frac{1}{2} \nabla_{\mu} \frac{1}{T^{2}} \\
& =\frac{1}{T^{2}}\left(A_{\mu}-\frac{1}{T} \nabla_{\mu} T\right) .
\end{aligned}
$$

One can recognize in this expression the four-vector which the heat flow $q^{\mu}$ is proportional to in the first-order dissipative hydrodynamics. Hence, we can say that the ideal relativistic Euler equation amounts to the statement that the first-order dissipative heat flow is parallel to the first-order dissipative current proportional to $\nabla \xi$. For an uncharged fluid, it simply states that at the first order in the gradient expansion, this dissipative current vanishes.

We can use (63) to obtain $D \xi$ as a function of $D \beta^{2}$ and plug into the first equation of (64), which then becomes

$$
\begin{aligned}
& {\left[\frac{\partial}{\partial \beta^{2}}\left(\left(\beta^{2}\right)^{3 / 2} \frac{\partial p}{\partial \beta^{2}}\right)-\frac{\left(\frac{\partial^{2} p}{\partial \beta^{2} \partial \xi}\right)^{2}\left(\beta^{2}\right)^{3 / 2}}{\frac{\partial^{2} p}{\partial \xi^{2}}}\right.} \\
& \left.-\frac{\frac{\partial p}{\partial \xi} \frac{\partial^{2} p}{\partial \beta^{2} \partial \xi}}{\frac{\partial^{2} p}{\partial \xi^{2}}} \sqrt{\beta^{2}}-\frac{\left(\frac{\partial p}{\partial \xi}\right)^{2}}{4 \sqrt{\beta^{2}} \frac{\partial^{2} p}{\partial \xi^{2}}}\right] D \beta^{2} \\
& +\left[\frac{\partial p}{\partial \beta^{2}} \beta^{2}-\frac{\frac{\partial p}{\partial \xi}\left(\beta^{2} \frac{\partial^{2} p}{\partial \beta^{2} \partial \xi}+\frac{1}{2} \frac{\partial p}{\partial \xi}\right)}{\frac{\partial^{2} p}{\partial \xi^{2}}}\right] \nabla \cdot \beta=0 .
\end{aligned}
$$

This formula allows one to obtain the derivative of $\beta^{2}$ along the flow as a function of $\nabla \cdot \beta=(1 / T) \nabla \cdot u$. Similarly, one can obtain the transverse gradient of $\xi$ as a function of the derivatives of $\beta$ through the (64). It should be kept in mind that these relations hold up to terms of the second order in the gradients. They can be used to eliminate some 
of the gradients in the first-order expansion of the stressenergy tensor, or, better, to replace some of the first-order gradients with transverse gradients of the $\beta$ field plus further corrections of the second order.

\subsection{Dissipative hydrodynamics in the $\beta$ frame}

Relativistic dissipative hydrodynamics has been the subject of intense investigations over the past decade [35-44] and an exhaustive discussion is well beyond the scope of this work. Herein, we confine ourselves to show that the $\beta$ frame is best suited to approach dissipative relativistic hydrodynamics as a gradient expansion. The main reason thereof has been mentioned in Sect. 5, that is, Eq. (32) expressing the density operator as a function of the present "time" local equilibrium operator and an integral of the gradients of the $\beta$ and $\xi$ fields. The expansion has been briefly outlined in Sect. 5 for the stress-energy tensor, but it can be extended to any observable.

We are now going to show that indeed, in the familiar first-order (Navier-Stokes) dissipative hydrodynamics, the transverse gradients of the velocity field and of the temperature can be re-expressed in terms of the gradients of $\beta$. We have already shown [see Eqs. $(65,66])$ that the combination

$\left(A_{\mu}-\frac{1}{T} \nabla_{\mu} T\right)$

appearing in the familiar form of the heat current vector can be rewritten in a compact way in terms of the gradients of $\beta$. Similarly, the transverse gradients of the velocity field $\nabla^{\mu} u^{v}$ can be written as follows:

$$
\begin{aligned}
\nabla_{\mu} u^{\nu} & =\nabla_{\mu} \frac{\beta^{\nu}}{\sqrt{\beta^{2}}}=\beta^{\nu}\left(-\frac{1}{2}\right)\left(\beta^{2}\right)^{-3 / 2} \nabla_{\mu} \beta^{2}+\frac{1}{\sqrt{\beta^{2}}} \nabla_{\mu} \beta^{\nu} \\
& =\frac{1}{\sqrt{\beta^{2}}}\left(-\frac{\beta^{\nu} \beta^{\rho}}{\beta^{2}} \nabla_{\mu} \beta_{\rho}+\nabla_{\mu} \beta^{\nu}\right)=\frac{1}{\sqrt{\beta^{2}}} \Delta^{\rho \nu} \nabla_{\mu} \beta_{\rho},
\end{aligned}
$$

where we have used the definition (39). Hence, the NavierStokes shear term can be fully expressed in terms of the inverse temperature four-vector. Likewise, it is easy to show the expansion term,

$\nabla_{\mu} u^{\mu}=\frac{1}{\sqrt{\beta^{2}}} \nabla_{\mu} \beta^{\mu}$.

\section{Conclusions}

We conclude with a short recapitulation of the main findings of this work.

- The notion of relativistic local thermodynamical equilibrium (LTE) can be defined independently of kinetic theory, in a form which is suitable for a strongly interacting fluid.
- The local thermodynamical equilibrium notion is, by construction, frame dependent. There is a preferred frame for it, the one where the basic thermodynamics relations take on the simplest form, the one we have called the $\beta$ frame; the $\beta$ frame is the frame when expansions from LTE are to be carried out.

- Physically, the $\beta$ four-vector direction is identified by the four-velocity of an idealized relativistic thermometer at equilibrium with the system.

- The $\beta$ frame has many interesting features in relativistic hydrodynamics, both ideal and dissipative. The $\beta$ fourvector and the other intensive parameter $\xi=\mu / T$ are the solutions of Eq. (15) for a non-vortex $\beta$ field, or, in general, of Eq. (26).

- The $\beta$ frame in general differs from both the Eckart and the Landau frames. It differs from those frames in situations where a local acceleration is present, like in a rotating fluid. The local acceleration or rotation provides a new independent macroscopic scale which introduces quadratic corrections to the stress-energy tensor.

Furthermore, we have seen that the familiar ideal hydrodynamic equations of motion can be written in a form where $\beta$ and $\xi$ are the five unknown fields. Also, first-order dissipative hydrodynamics can be written in a form where the gradients are, again, only those of $\beta$ and $\xi$. It would be very interesting to extend the Israel-Stewart theory of causal hydrodynamics in terms of these fields and assess the stability of the equations.

Acknowledgments L.T. acknowledges financial support from the Polish National Science Center Grant No. DEC-2012/06/A/ST2/00390.

Open Access This article is distributed under the terms of the Creative Commons Attribution 4.0 International License (http://creativecomm ons.org/licenses/by/4.0/), which permits unrestricted use, distribution, and reproduction in any medium, provided you give appropriate credit to the original author(s) and the source, provide a link to the Creative Commons license, and indicate if changes were made.

Funded by SCOAP ${ }^{3}$.

\section{Appendix A: Stress-energy tensor for the free scalar field}

The Klein-Gordon equation of the real scalar field in cylindrical coordinates with Dirichlet boundary conditions $\widehat{\psi}(R)=0$ has the eigenfunctions:

$f_{\mathbf{n}}=C_{\mathbf{n}} J_{M}\left(p_{T} r\right) \exp \left[-i\left(\varepsilon_{\mathbf{n}} t-p_{L} z-M \phi\right)\right]$,

where $p_{L}$ is a continuous longitudinal momentum, $M$ is the integer angular momentum quantum number, and the (discrete) transverse momenta $p_{T}(M, l)$ with $l=0,1, \ldots$ are 
the solutions of the boundary condition equation:

$J_{M}\left(p_{T} R\right)=0$.

In the above two equations, $J_{M}$ is the Bessel function of integer order $M$. The quantity $\mathbf{n}=\left(p_{L}, M, l(M)\right)$ is the vector of quantum numbers, and the energy $\varepsilon_{\mathbf{n}}$ and the normalization coefficient $C_{\mathbf{n}}$ read

$\varepsilon_{\mathbf{n}}=\sqrt{m^{2}+p_{z}^{2}+p_{T}^{2}} \quad C_{\mathbf{n}}^{2}=\frac{1}{(2 \pi)^{2} \varepsilon_{\mathbf{n}} R^{2} J_{M}^{\prime 2}\left(p_{T} R\right)}$.

The eigenfunctions $f_{\mathbf{n}}$ are orthogonal:

$$
\begin{aligned}
& \int \mathrm{d}^{3} \mathrm{x} f_{\mathbf{n}}^{*} f_{\mathbf{n}^{\prime}}=C_{\mathbf{n}} C_{\mathbf{n}^{\prime}} \int \mathrm{d}^{3} \mathrm{x} J_{M}\left(p_{T} r\right) J_{M^{\prime}}\left(p_{T}^{\prime} r\right) \exp \\
& \quad \times\left\{i\left[\left(\varepsilon_{\mathbf{n}}-\varepsilon_{\mathbf{n}^{\prime}}\right) t-\left(p_{L}-p_{L}^{\prime}\right) z-\left(M-M^{\prime}\right) \phi\right]\right\} \\
& =C_{\mathbf{n}} C_{\mathbf{n}^{\prime}}(2 \pi)^{2} \delta\left(p_{L}-p_{L}^{\prime}\right) \delta_{M, M^{\prime}} \int_{0}^{R} \mathrm{~d} r r J_{M}\left(p_{T} r\right) J_{M}\left(p_{T}^{\prime} r\right) \exp \\
& \quad \times\left[i\left(\varepsilon_{\mathbf{n}}-\varepsilon_{\mathbf{n}^{\prime}}\right) t\right]=C_{\mathbf{n}}^{2}(2 \pi)^{2} \frac{R^{2}}{2} J_{M}^{\prime}\left(p_{T} R\right)^{2} \delta_{\mathbf{n}, \mathbf{n}^{\prime}},
\end{aligned}
$$

where in the last equality we took advantage of the orthogonality relations of Bessel functions and

$\delta_{\mathbf{n}, \mathbf{n}^{\prime}} \equiv \delta\left(p_{L}-p_{L}^{\prime}\right) \delta_{M M^{\prime}} \delta_{l l^{\prime}}$.

The full orthogonality relations can be rewritten in the more compact form by using the normalization in Eq. (71):

$\int \mathrm{d}^{3} \mathrm{x} f_{\mathbf{n}}^{*} f_{\mathbf{n}^{\prime}}=\frac{1}{2 \varepsilon_{\mathbf{n}}} \delta_{\mathbf{n}, \mathbf{n}^{\prime}}$.

Another useful relation is

$$
\begin{aligned}
& \int \mathrm{d}^{3} \mathrm{x} f_{\mathbf{n}}^{*} f_{\mathbf{n}^{\prime}}^{*}=C_{\mathbf{n}} C_{\mathbf{n}^{\prime}} \int \mathrm{d}^{3} \mathrm{x} J_{M}\left(p_{T} r\right) J_{M^{\prime}}\left(p_{T}^{\prime} r\right) \exp \\
& \times\left\{i\left[\left(\varepsilon_{\mathbf{n}}+\varepsilon_{\mathbf{n}^{\prime}}\right) t-\left(p_{L}+p_{L}^{\prime}\right) z-\left(M+M^{\prime}\right) \phi\right]\right\} \\
& =C_{\mathbf{n}} C_{\mathbf{n}^{\prime}}(2 \pi)^{2} \delta\left(p_{L}+p_{L}^{\prime}\right) \delta_{M,-M^{\prime}} \\
& \quad \times \int_{0}^{R} \mathrm{~d} r r J_{M}\left(p_{T} r\right) J_{M^{\prime}}\left(p_{T}^{\prime} r\right) \exp \left[i\left(\varepsilon_{\mathbf{n}}+\varepsilon_{\mathbf{n}^{\prime}}\right) t\right] \\
& =\frac{1}{2 \varepsilon_{\mathbf{n}}}(-1)^{M} \exp \left(2 i \varepsilon_{\mathbf{n}} t\right) \delta_{\mathbf{n}^{\prime}, \tilde{\mathbf{n}}},
\end{aligned}
$$

where $\tilde{\mathbf{n}}=\left(-p_{L},-M, l\right)^{4}$, and in the last equality we used the $J_{-M}=(-1)^{M} J_{M}$ relation among the integer Bessel functions.

The field operator reads

$$
\widehat{\psi}(x)=\sum_{\mathbf{n}}\left[f_{\mathbf{n}} a_{\mathbf{n}}+f_{\mathbf{n}}^{*} a_{\mathbf{n}}^{\dagger}\right] .
$$

From Eqs. (73) and (74) and the canonical equal time commutation relations,

$[\widehat{\psi}(t, \mathbf{x}), \widehat{\psi}(t, \mathbf{y})]=\left[\partial_{t} \widehat{\psi}(t, \mathbf{x}) \equiv \widehat{\Pi}(t, \mathbf{x}), \widehat{\Pi}(t, \mathbf{y})\right]=0$

\footnotetext{
${ }^{4}$ It is important to note that $\varepsilon_{\tilde{\mathbf{n}}}=\varepsilon_{\mathbf{n}}$ and $C_{\tilde{\mathbf{n}}}=C_{\mathbf{n}}$.
}

$[\widehat{\psi}(t, \mathbf{x}), \widehat{\Pi}(t, \mathbf{y})]=i \delta^{3}(\mathbf{x}-\mathbf{y})$,

the commutation relations between creation and annihilation operators $a_{\mathbf{n}}^{\dagger}, a_{\mathbf{n}}$ follow:

$\left[a_{\mathbf{n}}, a_{\mathbf{n}^{\prime}}\right]=0 \quad\left[a_{\mathbf{n}}^{\dagger}, a_{\mathbf{n}^{\prime}}^{\dagger}\right]=0 \quad\left[a_{\mathbf{n}}, a_{\mathbf{n}^{\prime}}^{\dagger}\right]=\delta_{\mathbf{n}, \mathbf{n}^{\prime}}$.

Reasoning as in Ref. [26], one can readily show that

$\left\langle a_{\mathbf{n}} a_{\mathbf{n}^{\prime}}\right\rangle=\left\langle a_{\mathbf{n}}^{\dagger} a_{\mathbf{n}^{\prime}}^{\dagger}\right\rangle=0 \quad\left\langle a_{\mathbf{n}}^{\dagger} a_{\mathbf{n}^{\prime}}\right\rangle=\frac{1}{\mathrm{e}^{\left(\varepsilon_{\mathbf{n}}-M \omega\right) / T_{0}}-1} \delta_{\mathbf{n}, \mathbf{n}^{\prime}}$,

where in the last term one can recognize the typical Bose statistics mean occupation number, henceforth denoted as $n_{B}$ :

$n_{B} \equiv \frac{1}{\mathrm{e}^{\left(\varepsilon_{\mathbf{n}}-M \omega\right) / T_{0}}-1}$.

We can now calculate the projections of the mean stressenergy tensor $\langle: \widehat{T}:\rangle$ with $\widehat{T}$ like in Eq. (57), in the basis $\{u, n, k, \tau\}$. First, we calculate the mean value $\langle: \widehat{\mathcal{L}}:\rangle$ of the Lagrangian density in Ref. (57); for this purpose, one needs derivatives of the field:

$$
\begin{aligned}
& \partial_{r} \widehat{\psi}=\sum_{\mathbf{n}}\left[\left(\partial_{r} f_{\mathbf{n}}\right) a_{\mathbf{n}}+\left(\partial_{r} f_{\mathbf{n}}^{*}\right) a_{\mathbf{n}}^{\dagger}\right] \\
& \partial_{z} \widehat{\psi}=\sum_{\mathbf{n}}\left(i p_{L}\right)\left[f_{\mathbf{n}} a_{\mathbf{n}}-f_{\mathbf{n}}^{*} a_{\mathbf{n}}^{\dagger}\right] \\
& \partial_{\varphi} \widehat{\psi}=\sum_{\mathbf{n}}(i M)\left[f_{\mathbf{n}} a_{\mathbf{n}}-f_{\mathbf{n}}^{*} a_{\mathbf{n}}^{\dagger}\right] .
\end{aligned}
$$

Setting the above expansions in the Lagrangian in Eq. (57), one obtains

$$
\begin{aligned}
\langle: \widehat{\mathcal{L}}:\rangle= & \frac{1}{2}\left\langle:\left(\partial_{t} \widehat{\psi}\right)^{2}-\left(\partial_{x} \widehat{\psi}\right)^{2}-\left(\partial_{y} \widehat{\psi}\right)^{2}-\left(\partial_{z} \widehat{\psi}\right)^{2}-m^{2} \widehat{\psi}^{2}:\right\rangle \\
= & \frac{1}{2}\left\langle:\left(\partial_{t} \widehat{\psi}\right)^{2}-\left(\partial_{r} \widehat{\psi}\right)^{2}-\frac{1}{r^{2}}\left(\partial_{\varphi} \widehat{\psi}\right)^{2}-\left(\partial_{z} \widehat{\psi}\right)^{2}-m^{2} \widehat{\psi}^{2}:\right\rangle \\
= & \sum_{\mathbf{n}} n_{B}\left\{\varepsilon_{\mathbf{n}}^{2}\left|f_{\mathbf{n}}\right|^{2}-\left|\partial_{r} f_{\mathbf{n}}\right|^{2}\right. \\
& \left.-\frac{M^{2}}{r^{2}}\left|f_{\mathbf{n}}\right|^{2}-p_{L}^{2}\left|f_{\mathbf{n}}\right|^{2}-m^{2}\left|f_{\mathbf{n}}\right|^{2}\right\} \\
= & \sum_{\mathbf{n}} n_{B}\left\{\left(p_{T}^{2}-\frac{M^{2}}{r^{2}}\right)\left|f_{\mathbf{n}}\right|^{2}-\left|\partial_{r} f_{\mathbf{n}}\right|^{2}\right\} .
\end{aligned}
$$

Every projection involving one $k$ is vanishing as $k \cdot \partial=\partial_{z}$ involves a multiplication of each term within the sum $\sum_{\mathbf{n}}$ by $p_{L}$. On the other hand, the $k \cdot T \cdot k$ diagonal term reads

$$
\begin{aligned}
& k \cdot T \cdot k=\left\langle:\left(\partial_{z} \widehat{\psi}\right)^{2}+\mathcal{L}:\right\rangle \\
& \quad=\sum_{\mathbf{n}} n_{B}\left\{2 p_{L}^{2}\left|f_{\mathbf{n}}\right|^{2}+\left(p_{T}^{2}-\frac{M^{2}}{r^{2}}\right)\left|f_{\mathbf{n}}\right|^{2}-\left|\partial_{r} f_{\mathbf{n}}\right|^{2}\right\} .
\end{aligned}
$$


Similarly, for the projections along $n$, the off-diagonal $\hat{r} \cdot T \cdot \hat{\tau}$ and $\hat{r} \cdot T \cdot u$ terms vanish because

$$
\begin{aligned}
& \left.\left\langle: \partial_{(t} \widehat{\psi} \partial_{r}\right) \widehat{\psi}:\right\rangle=\sum_{\mathbf{n}} n_{B}\left\{-i \varepsilon_{\mathbf{n}} f_{\mathbf{n}}^{*}\left(\partial_{r} f_{\mathbf{n}}\right)+i \varepsilon_{\mathbf{n}} f_{\mathbf{n}}^{*}\left(\partial_{r} f_{\mathbf{n}}\right)\right\}=0 \\
& \left\langle: \partial_{(\varphi} \widehat{\psi} \partial_{r)} \widehat{\psi}:\right\rangle=\sum_{\mathbf{n}} n_{B}\left\{i M f_{\mathbf{n}}\left(\partial_{r} f_{\mathbf{n}}^{*}\right)-i M f_{\mathbf{n}}^{*}\left(\partial_{r} f_{\mathbf{n}}\right)\right\}=0,
\end{aligned}
$$

taking into account that $f_{\mathbf{n}}\left(\partial_{r} f_{\mathbf{n}}^{*}\right)$ is real. On the other hand,

$$
\begin{aligned}
& \hat{r} \cdot T \cdot \hat{r}=\left\langle:\left(\partial_{r} \widehat{\psi}\right)^{2}+\widehat{\mathcal{L}}:\right\rangle \\
& \quad=\sum_{\mathbf{n}} n_{B}\left[\left|\partial_{r} f_{\mathbf{n}}\right|^{2}+\left(p_{T}^{2}-\frac{M^{2}}{r^{2}}\right)\left|f_{\mathbf{n}}\right|^{2}\right] .
\end{aligned}
$$

Using the relations

$u \cdot \partial=\gamma \partial_{t}+\gamma v \frac{1}{r} \partial_{\varphi}=\gamma \partial_{t}+\gamma \omega \partial_{\varphi} \quad \hat{\tau} \cdot \partial=\gamma v \partial_{t}+\gamma \frac{1}{r} \partial_{\varphi}$,

we can calculate the diagonal projections onto $u$ and $\hat{\tau}$ :

$$
\begin{aligned}
u \cdot T & \cdot u=\left\langle: \gamma^{2}\left[\left(\partial_{t} \widehat{\psi}\right)^{2}+\omega^{2}\left(\partial_{\varphi} \widehat{\psi}\right)^{2}+2 \omega\left(\partial_{(t} \widehat{\psi} \partial_{\varphi} \widehat{\psi}\right)\right]-\mathcal{L}:\right\rangle \\
= & \sum_{\mathbf{n}} n_{B}\left\{2 \gamma^{2}\left[\varepsilon_{\mathbf{n}}^{2}+M^{2} \omega^{2}-2 \varepsilon_{\mathbf{n}} M \omega\right]\left|f_{\mathbf{n}}\right|^{2}\right. \\
& \left.-\left(p_{T}^{2}-\frac{M^{2}}{r^{2}}\right)\left|f_{\mathbf{n}}\right|^{2}+\left|\partial_{r} f_{\mathbf{n}}\right|^{2}\right\} \\
= & \sum_{\mathbf{n}} n_{B}\left\{2 \gamma^{2}\left[\varepsilon_{\mathbf{n}}-M \omega\right]^{2}\left|f_{\mathbf{n}}\right|^{2}\right. \\
& \left.-\left(p_{T}^{2}-\frac{M^{2}}{r^{2}}\right)\left|f_{\mathbf{n}}\right|^{2}+\left|\partial_{r} f_{\mathbf{n}}\right|^{2}\right\} \\
\hat{\tau} \cdot T & \cdot \hat{\tau}=\left\langle: \gamma^{2}\left[v^{2}\left(\partial_{t} \widehat{\psi}\right)^{2}+\left(\frac{1}{r} \partial_{\varphi} \widehat{\psi}\right)^{2}+2 \omega\left(\partial_{(t} \widehat{\psi} \partial_{\varphi} \widehat{\psi}\right)\right]+\mathcal{L}:\right\rangle \\
= & \sum_{\mathbf{n}} n_{B}\left\{2 \gamma^{2}\left[\varepsilon_{\mathbf{n}}^{2} \omega^{2} r^{2}+\frac{M^{2}}{r^{2}}-2 \varepsilon_{\mathbf{n}} M \omega\right]\left|f_{\mathbf{n}}\right|^{2}\right. \\
& \left.+\left(p_{T}^{2}-\frac{M^{2}}{r^{2}}\right)\left|f_{\mathbf{n}}\right|^{2}-\left|\partial_{r} f_{\mathbf{n}}\right|^{2}\right\} \\
= & \sum_{\mathbf{n}} n_{B}\left\{2 \gamma^{2}\left[\varepsilon_{\mathbf{n}} v-\frac{M}{r}\right]^{2}\left|f_{\mathbf{n}}\right|^{2}\right. \\
& \left.+\left(p_{T}^{2}-\frac{M^{2}}{r^{2}}\right)\left|f_{\mathbf{n}}\right|^{2}-\left|\partial_{r} f_{\mathbf{n}}\right|^{2}\right\},
\end{aligned}
$$

as well as the non-diagonal term:

$$
\begin{aligned}
& u \cdot T \cdot \hat{\tau}=\left\langle: \gamma^{2}\left\{v\left[\left(\partial_{t} \widehat{\psi}\right)^{2}+\frac{1}{r^{2}}\left(\partial_{\varphi} \widehat{\psi}\right)^{2}\right]\right.\right. \\
& \left.\left.\quad+\frac{1}{r}\left(1+v^{2}\right) \partial_{(t} \widehat{\psi} \partial_{\varphi)} \widehat{\psi}\right\}:\right\rangle \\
& =2 \gamma^{2} \sum_{\mathbf{n}} n_{B}\left\{\omega r\left(\varepsilon_{\mathbf{n}}^{2}+\frac{M^{2}}{r^{2}}\right)-\left(1+\omega^{2} r^{2}\right) \frac{\varepsilon_{\mathbf{n}} M}{r}\right\}\left|f_{\mathbf{n}}\right|^{2},
\end{aligned}
$$

or, writing explicitly the $\left|f_{\mathbf{n}}\right|^{2}$ function

$u \cdot T \cdot \hat{\tau}=2 \gamma^{2} \sum_{\mathbf{n}} \frac{J_{M}^{2}\left(p_{T} r\right)}{(2 \pi)^{2} \varepsilon_{\mathbf{n}} R^{2} J_{M}^{\prime 2}\left(p_{T} R\right)} \frac{1}{\mathrm{e}^{\left(\varepsilon_{\mathbf{n}}-M \omega\right) / T_{0}}-1}$

$$
\times\left[\omega r\left(\varepsilon_{\mathbf{n}}^{2}+\frac{M^{2}}{r^{2}}\right)-\left(1+\omega^{2} r^{2}\right) \frac{\varepsilon_{\mathbf{n}} M}{r}\right] .
$$

\section{Appendix B: Thermodynamic relations and change of frame}

In this section we show how the basic thermodynamic relation (17) between proper entropy density, proper energy, and charge density, pressure, and temperature is modified by a change of frame. We first consider the familiar global equilibrium case, where $\beta$ is a constant four-vector. It should be first pointed out that in the case of global equilibrium with constant $\beta$, its direction coincides with both the Landau and the Eckart four-velocities, that is, there exists one four-vector to which all physical vectors are parallel $[24,25]$. According to our reasoning in Sect. 2, in this case the entropy current $s^{\mu}$ reads, as is well known [24,25],

$s^{\mu}=p \beta^{\mu}+T^{\mu \nu} \beta_{v}-\xi j^{\mu}$.

Note that $T^{\mu \nu}=T_{\mathrm{LE}}^{\mu \nu}$ and $j^{\mu}=j_{\mathrm{LE}}^{\mu}$, being at global equilibrium; also note that the extra term $s_{T}^{\mu}$ in Eq. (14) is now missing, because the total entropy is conserved, thus it should be the same for any three-dimensional spacelike hypersurface chosen to integrate the entropy current. Let us now project onto an arbitrary frame $u$ the above expression, replacing first $T^{\mu \nu}$ with its expression at global equilibrium $(\rho+p) \hat{\beta}^{\mu} \hat{\beta}^{\nu}-p g^{\mu \nu}$ and likewise for $j^{\mu}=q \hat{\beta}^{\mu}$ :

$s^{\mu} u_{\mu} \equiv s_{u}=p(\beta \cdot u)+\rho(\beta \cdot u)-\frac{\xi}{\sqrt{\beta^{2}}} q(\beta \cdot u)$.

The functions $p\left(\beta^{2}, \xi\right), \rho\left(\beta^{2}, \xi\right)$, and $q\left(\beta^{2}, \xi\right)$ are, by definition, the usual thermodynamic functions, with $\beta^{2} \equiv 1 / T^{2}$ and $\xi=\mu / T$. Now we recall that $\beta \cdot u$ is the inverse temperature marked by the thermometer moving at speed $u$, say $T_{u}$; hence the Eq. (82) can be rewritten as

$T_{u} s_{u}=p+\rho-\mu q$.

However, the functions $p, \rho, q$ are not formally the same thermodynamic functions of $T$ as of $T_{u}$. The difference between the two can be obtained by setting $\hat{\beta}=u+\delta u$, whence

$\delta u \cdot u=-(\delta u)^{2}$,

so that

$\frac{1}{T_{u}}=\beta \cdot u=\sqrt{\beta^{2}}(\hat{\beta} \cdot u)=\frac{1}{T}\left[1-(\delta u)^{2}\right]$.

Thus, the difference between the thermodynamic relation in the two frames is of the second order in the difference between velocity fields.

Of course, in the global equilibrium case with constant $\beta$, as has been mentioned, there is no difference between different frames. However, in LTE, the choice of the frame 
changes the basic thermodynamic relation by terms of the second order in the difference between velocities.

In the case of global equilibrium with rotation, where, as we have shown, the $\beta$ and the Landau frames do not coincide, the basic thermodynamic relation cannot be the simplest one (20) in both frames. If $\phi^{\mu}=p \beta^{\mu}$ as in the previous case, then the $\beta$ frame is the one where (20) holds.

\section{References}

1. R. Baier, P. Romatschke, D.T. Son, A.O. Starinets, M.A. Stephanov, JHEP 0804, 100 (2008)

2. P. Kovtun, D.T. Son, A.O. Starinets, Phys. Rev. Lett. 94, 111601 (2005)

3. P.F. Kolb, U.W. Heinz, P. Huovinen, K.J. Eskola, K. Tuominen, Nucl. Phys. A 696, 197 (2001)

4. J.-Y. Ollitrault, Eur. J. Phys. 29, 275 (2008)

5. U. Heinz, R. Snellings, Ann. Rev. Nucl. Part. Sci. 63, 123 (2013)

6. R. Baier, P. Romatschke, U.A. Wiedemann, Phys. Rev. C 73, 064903 (2006)

7. P. Huovinen, P.V. Ruuskanen, Ann. Rev. Nucl. Part. Sci. 56, 163 (2006)

8. L.P. Csernai, J.I. Kapusta, L.D. McLerran, Phys. Rev. Lett. 97, 152303 (2006)

9. W. Florkowksi, Phenomenology of Ultra-Relativistic Heavy Ion Collisions (World Scientific, Singapore, 2010)

10. P. Bozek, W. Broniowski, Acta Phys. Polon. B 45(7), 1337 (2014). arXiv:1403.6042 [nucl-th]

11. K. Damle, S. Sachdev, Phys. Rev. B 56, 8714 (1997)

12. S.A. Hartnoll, P.K. Kovtun, M. Müller, S. Sachdev, Phys. Rev. B 76, 144502 (2007)

13. P. Kovtun, C. Herzog, S. Sachdev, D.T. Son, Phys. Rev. D 75, 085020 (2007)

14. L. Fritz, J. Schmalian, M. Muller, S. Sachdev, Phys. Rev. B 78, 085416 (2008)

15. A. Adams, L.D. Carr, T. Schäfer, P. Steinberg, J.E. Thomas, New J. Phys. 14, 115009 (2012)

16. J. Casalderrey-Solana, H. Liu, D. Mateos, K. Rajagopal, U.A. Wiedemann, arXiv:1101.0618 [hep-th]
17. G.S. Denicol, E. Molnár, H. Niemi, D.H. Rischke, Eur. Phys. J. A 48, 170 (2012)

18. S.R. De Groot, W.A. van Leeuwen, C.H.G. van Weert, Relativistic Kinetic Theory (North Holland, Amsterdam, 1980)

19. R. Balian, From Microphysics to Macrophysics (Springer, Heidelberg, 2007)

20. P. Ván, T.S. Biró, in Proceedings of the 12th Joint European Thermodynamics Conference, Cartolibreria SNOOPY, 2013, ed. by M. Pilotelli, G.P. Beretta, pp. 546-551. arXiv:1305.3190 [gr-qc]

21. D.N. Zubarev, A.V. Prozorkevich, S.A. Smolyanskii, Theoret. Math. Phys. 40, 821 (1979)

22. F. Becattini, Phys. Rev. Lett. 108, 244502 (2012)

23. T. Chrobok, H.H. von Borzeszkowski, Gen. Rel. Grav. 38, 397 (2006)

24. W. Israel, Ann. Phys. 100, 310 (1976)

25. W. Israel, J.M. Stewart, Ann. Phys. 118, 341 (1979)

26. F. Becattini, L. Tinti, Phys. Rev. D 84, 025013 (2011)

27. A. Einstein, Jahrb. Rad. u. Elektr. 4, 411 (1907)

28. M. Planck, Ann. der Phys. 26, 1 (1908)

29. D.N. Zubarev, Sov. Phys. Doklady 10, 850 (1966)

30. D.N. Zubarev, M.V. Tokarchuk, Theor. Math. Phys. 88, 876 (1992) [Teor. Mat. Fiz. 88N2, 286 (1991)]

31. A. Hosoya, M. Sakagami, M. Takao, Ann. Phys. 154, 229 (1984)

32. G.D. Moore, K.A. Sohrabi, JHEP 1211, 148 (2012)

33. P. Kovtun, J. Phys. A 45, 473001 (2012)

34. A. Muronga, Phys. Rev. Lett. 88, 062302 (2002) [Erratum-ibid. 89, 159901 (2002)]

35. P. Huovinen, D. Molnar, Phys. Rev. C 79, 014906 (2009)

36. U.W. Heinz, H. Song, A.K. Chaudhuri, Phys. Rev. C 73, 034904 (2006)

37. A. Muronga, Heavy Ion Phys. 15, 337 (2002)

38. A. Muronga, Phys. Rev. C 69, 034903 (2004)

39. G.S. Denicol, T. Kodama, T. Koide, P. Mota, J. Phys. G 35, 115102 (2008)

40. P. Romatschke, Int. J. Mod. Phys. E 19, 1 (2010)

41. P. Romatschke, Class. Q. Grav. 27, 025006 (2010)

42. K. Jensen, M. Kaminski, P. Kovtun, R. Meyer, A. Ritz, A. Yarom, Phys. Rev. Lett. 109, 101601 (2012)

43. P. Van, T.S. Biro, Phys. Lett. B 709, 106 (2012)

44. J. Bhattacharya, S. Bhattacharyya, M. Rangamani, JHEP 1302, 153 (2013) 